\title{
Changes in phytoplankton community structure at three touristic sites at western Alexandria Beach.
}

\author{
Nihal G. Shams El Din and Ahmed M. Abel Halim \\ National Institute of Oceanography and Fisheries, Alexandria, Egypt
}

\begin{abstract}
The phytoplankton abundance, chlorophyll- $a$ and diversity cycle in the Mediterranean sea water, west of Alexandria (Naubaria, reference site), El Mohandessin, Marakia and Marabella tourism villages were studied during the perivd from March 2005 to Jan. 2006 and the results were discussed in relation to some environmental physico-chemical parameters. A total of 208 taxa were recorded in the study area and characterized by different ecological affinities extending from typical marine forms (107 taxa), to freshwater euryhaline forms (101 taxa). The Naubaria site was the most diversified community (176 taxa) followed by El Mohandessin village site (90 taxa), Marakia village site ( 83 taxa) and Marabella village site (54 taxa). Bacillariophyceae was the predominant group in the study area except in Marabella village site, where Chlorophyceae was predominant in September $(99.53 \%)$. Few species were responsible for abundance peaks, namely: Cyclotella kütziginiana, Rhizosolenia fragellissima (July), Rhizosolenia setigera (September), Skeletonema costatum (November) in Naubaria site; Skeletonema costatum (November) in El Mohandessin site; Rhizosolenia setigera (September) in Marakia site; Carteria sp. (September) in Marabella site. Chlorophyll- $a$ varied greatly from an average of $0.77 \mu \mathrm{g} / \mathrm{L}$ at Marabella site to $2.66 \mu \mathrm{g} / \mathrm{L}$ at Naubaria site, classifying Naubaria and ElMohandessin sites between mesotrophic to oligotrophic status and Marakia and Marabella sites as oligotrophic. Species diversity had a negative effect on phytoplankton abundance, ranging between 0.53 and 2.99 nats at Naubaria site, 0.13 and 2.39 nats at El Mohandessin site, 0.56 and 2.54 nats at Marakia site, and between 0.03 and 2.79 nats at Marabella site. The blooms of few dominant species were accompanied with low diversity under the prevailing ecological conditions reflecting the effect of wastewater effluents discharged in Naubaria, El-Mohandessin and Marabella sites. Thus, they should be kept under investigation to control pollution, whereas the co-dominance of several species, accompanied with low nutrient concentrations at Marakia village site suggests free conditions.
\end{abstract}

Key words: Phytoplankton, community structure, Biodiversity, tourism, Alexandria

\section{INTRODUCTION}

During the past few decades, marine coastal area of Alexandria have been exposed to many environmental hazards (Abdallah et al., 1995; Zaghloul, 
1995; El-Sherif and Mikhail, 2003; Shams El Din and Dorgham, 2007). Significant anthropogenic input is mainly due to industrial activities and developed tourism villages. The coast is a receiver of effluents of domestic and industrial origin, which usually lead to environmental and health problems.

The west Naubaria Canal was constructed to collect agricultural drainage water from the cultivated land around south and south west of Alexandria. In 1986, it was connected to the Mediterranean Sea at the kilometer 21 of Alexandria-Matrouh Highway (Gharib and Dorgham 2003 and Abdel Aziz, 2005). It receives also industrial and domestic wastes. The canal is connected at certain part with El-omum Drain which discharges great amount of different types of waste water to Mex Bay.

Since the opening of Naubaria Canal, there have been few studies known to be carried out on the ecological and biological characteristics of its water. The first study was carried out through IMP (Environmental Impact Monitoring Program) by EEAA (Egyptian Environmental Affairs Agency) and focused on the hydrographic and eutrophication conditions. Phytoplankton and zooplankton densities were weekly investigated in the northern part of Naubaria Canal (Gharib and Dorgham, 2003; Abdel Aziz, 2005). On the other hand, there are no planktonic studies known to be carried out on the shore area of the nominated tourism villages (El Mohandessin, Marakia and Marabella). The present study is yet the first one dealing with the phytoplankton community and its relation to the physico-chemical characteristics. It aimed to evaluate the effect of tourism on the marine ecosystem at the three tourism villages, being a part of a project on studying the effect of industrial activities, marine transport and tourism on physico-chemical and biological characteristics of water and fisheries west of Alexandria.

\section{MATERIAL AND METHODS}

Water sampling was performed from 10 to 14 O'clock at morning bimonthly from March 2005 to Jannuary 2006 covering four sites along the western coast of Alexandria (Fig. 1).

\section{Study area:}

\section{A- Naubaria site (reference site):}

Naubaria canal is connected to the Mediterranean Sea at the kilometer 21 of Alexandria-Matrouh Highway, at latitude $31^{\circ} 09^{\prime} \mathrm{N}$ and longitude $29^{\circ} 71^{\prime}$ E. Two sampling sites (I\&II) were selected. The first one was chosen in the canal, whereas the second was $0.5 \mathrm{~m}$ far from the opening of the canal, lying in the sea. Two other stations (III\&IV) were selected. Samples were collected from the surface layer and near the bottom layer in front of the canal at the open Sea (at 2 and $4 \mathrm{~m}$ far from the opening of the canal, respectively).

\section{B- El-Mohandessin tourism village site:}

The village lies at $25 \mathrm{~km}$ west of Alexandria and adjacent to Sumid Petroleum Company and Sidi-Krir Power plant, at latitude $31^{\circ} 06^{\prime} \mathrm{N}$ and 
longitude $29^{\circ} 68^{\prime} \mathrm{E}$. It is subjected to thermal pollution, in addition to pollution as a result of wastewater discharge from tourism activities.

\section{C- Marakia tourism village site:}

The village lies at $50 \mathrm{~km}$ west of Alexandria at latitude $30^{\circ} 94^{\prime} \mathrm{N}$ and longitude $29^{\circ} 48^{\prime} \mathrm{E}$ and is subjected to wastewater effluent discharge.

\section{D- Marabella tourism village site:}

This village lies at $68 \mathrm{~km}$ west of Alexandria at latitude $30^{\circ} 88^{\prime} \mathrm{N}$ and longitude $39^{\circ} 34^{\prime} \mathrm{E}$ and is subjected to wastewater effluent discharge.

The samples were collected at the surface layer at the three touristic sites which are shallow areas and do not exceeded $3 \mathrm{~m}$ depth.

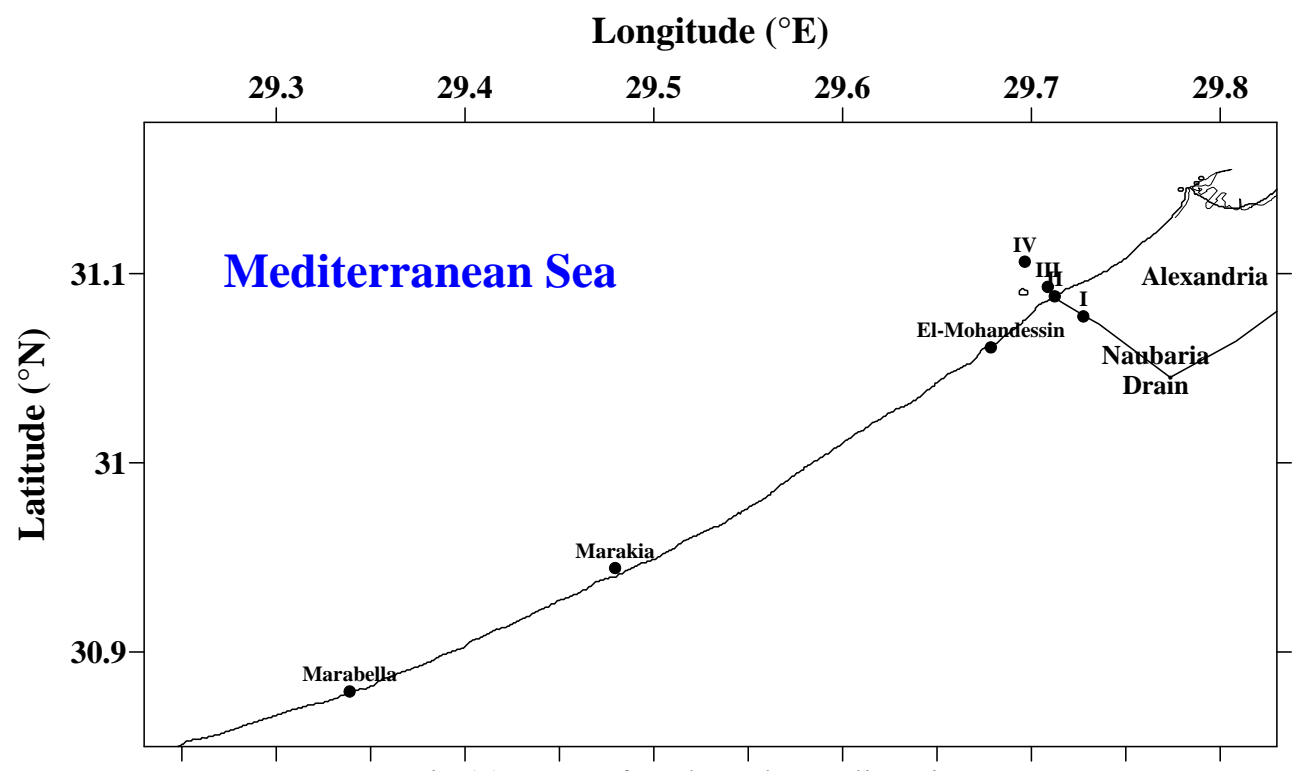

Fig (1): Area of study and sampling sites

\section{Physico-chemical study:}

Temperature and $\mathrm{pH}$ were measured in situ using protected thermometer $\left(0.01^{\circ} \mathrm{C}\right)$ and portable $\mathrm{pH}$ meter (Orion $\mathrm{pH}$-meter) respectively. Salinity was measured using Echman salinometer. Water transparency was determined by secchi disc. Dissolved oxygen (DO) was analysed according to the modified Winkler's method (APHA, 1995). The nutrient salts $\left(\mathrm{NO}_{2}, \mathrm{NO}_{3}, \mathrm{NH}_{4}, \mathrm{PO}_{4}\right.$ and $\mathrm{SiO}_{4}$ ) were determined colorimetrically according to the methods of Grasshoff (1976) using a UV-Visible spectrophotometer (Shimadzu double beam Model 150-02). Oxidizable organic matter (OOM) was estimated chemically according to Ellis et al., (1946).

\section{Phytoplankton study:}

Quantitative phytoplankton samples were collected at each site by a two liters capacity Niskin Bottle. The samples were preserved immediately in 4\% 
neutralized formalin, left 48 hours for settling of phytoplankton forms and then the supernatant water was decanted. Estimation of phytoplankton abundance was carried out by the sedimentation method (APHA, 1995). The phytoplankton species were identified, counted and expressed as unit/ liter (unit comprised: cells, filaments and colonies). Identification of algal taxa followed Peragallo \& Peragallo (1897-1908), Lebour (1925), Cupp (1943), Heurk (1962), Sournia $(1968,1986)$, Tikkanen (1986) and Mizuno (1990).

\section{Chlorophyll- $a$ :}

For estimation of total Chlorophyll- $a$, one liter of water sample was filtered by GFC filtration paper and algae were extracted with $10 \mathrm{ml}$ of $90 \%$ aqueous acetone solution and were determined according to APHA (1995). For estimation of chlorophyll concentration of phytoplankton $>20 \mu \mathrm{m}$ a water sample ( 1 liter) was filtered by a net of mesh size $20 \mu \mathrm{m}$ followed by the same steps of determination of total chlorophyll. Then nannoplankton biomass was calculated as the difference between the two estimations.

\section{Diversity index:}

Diversity indices of phytoplankton community was calculated according to Shannon and Weaver (1963), the equitability (evenness) according to Heip (1974), and species richness according to Margalef (1968) and Heip (1974).

\section{Naubaria canal site (reference site):}

\section{RESULTS}

Water temperature at Naubaria site showed temporal variations within the normal seasonal fluctuation $\left(12.57-30.00{ }^{\circ} \mathrm{C}\right.$ ) (Gharib and Dorgham, 2003) (Table 1). Surface water temperature was slightly warmer than near bottom layer in summer. The variations of water salinity in Naubaria site reflected the interaction between the freshwater from Naubaria Canal and the sea water (7.6739.72PSU) (Table 1). Station I, which was facing the inflow of freshwater had usually the lowest value (average 9.73 PSU). Salinity at the other stations reflected the effect of sea water (averages 33.4-39.00 PSU). On the other hand, surface water salinity was usually lower than that near bottom layer. The freshwater flow from Naubaria Canal carrying suspended matter is the major cause of water turbidity at St. I (average $38 \mathrm{~cm}$ ) and transparency increased seawards to reach $>120 \mathrm{~cm}$. The $\mathrm{pH}$ values lied on the alkaline side (7.95-8.92) (Table 1), with the lower values near bottom layer. Dissolved oxygen concentration lied between 4.16 and $14.95 \mathrm{mg} \mathrm{O}_{2} / \mathrm{L}$. The freshwater inflow from Naubaria Canal was enriched with nutrient salts and so St.I had usually the highest values of nutrient salts and the concentration decreased seawards. Ammonia ranged from nil to $16.88 \mu \mathrm{M} / \mathrm{L}$, nitrite from 0.00 to $6.40 \mu \mathrm{M} / \mathrm{L}$, nitrate from 0.00 to $69.17 \mu \mathrm{M} / \mathrm{L}$, phosphate from 0.00 to $23.04 \mu \mathrm{M} / \mathrm{L}$ and silicate from 0.11 to $105.74 \mu \mathrm{M} / \mathrm{L}$. The oxidizable organic matter showed wide variations from nil to $50.6 \mathrm{mg} / \mathrm{L}$ (Table 1). 
Table (1): Range and mean values of different hydrographic parameters in Naubaria, ElMohandessin, Marakia and Marabella sites during March 2005 - January 2006.

\begin{tabular}{|c|c|c|c|c|c|c|c|c|c|c|c|c|}
\hline & \multicolumn{3}{|c|}{ Naubaria site } & \multicolumn{3}{|c|}{ El-Mohandessin site } & \multicolumn{3}{|c|}{ Marakia site } & \multicolumn{3}{|c|}{ Marabella site } \\
\hline & \multicolumn{2}{|c|}{ Range } & \multirow{2}{*}{$\begin{array}{c}\text { Mean } \\
* * *\end{array}$} & \multicolumn{2}{|c|}{ Range } & \multirow{2}{*}{$\begin{array}{c}\text { Mean } \\
* * *\end{array}$} & \multicolumn{2}{|c|}{ Range } & \multirow{2}{*}{$\begin{array}{c}\text { Mean } \\
* * *\end{array}$} & \multicolumn{2}{|c|}{ Range } & \multirow{2}{*}{$\begin{array}{c}\text { Mean } \\
* * *\end{array}$} \\
\hline $\begin{array}{l}\text { Water temperature } \\
\qquad\left({ }^{\circ} \mathrm{C}\right)\end{array}$ & 12.57 & 30.0 & & 15.34 & 30.00 & & 15.56 & 30.00 & & 16.61 & 30.00 & \\
\hline Salinity (PSU) & 7.67 & 39.72 & 32.94 & 36.88 & 38.80 & 37.97 & 38.65 & 39.80 & 39.20 & 38.61 & 39.95 & 39.22 \\
\hline Transparency $(\mathrm{cm})$ & 10 & 1200 & 436 & 100 & 150 & 130 & 100 & 200 & 163 & 100 & 300 & 217 \\
\hline $\mathbf{p H}$ & 7.95 & 8.92 & $* * *$ & 8.08 & 8.24 & $* * *$ & 8.03 & 8.09 & $* * *$ & 7.98 & 8.27 & $* * *$ \\
\hline Dissolved oxygen (mg/L) & 4.16 & 14.95 & 7.78 & 4.01 & 13.57 & 7.99 & 5.19 & 13.57 & 8.57 & 4.25 & 19.55 & 10.07 \\
\hline $\mathrm{NH}_{4}(\mu \mathrm{M}) / \mathrm{L}$ & nil & 16.88 & 3.50 & 1.80 & 4.72 & 3.25 & 0.14 & 24.25 & 5.17 & 0.50 & 10.48 & 3.97 \\
\hline $\mathrm{NO}_{2}(\mu \mathrm{M}) / \mathrm{L}$ & nil & 6.40 & 0.90 & 0.00 & 1.70 & 0.71 & 0.00 & 0.30 & 0.11 & 0.00 & 1.03 & 0.32 \\
\hline $\mathrm{NO}_{3}(\mu \mathrm{M}) / \mathrm{L}$ & nil & 69.17 & 10.05 & 0.00 & 28.93 & 8.23 & 0.10 & 4.01 & 2.10 & 0.44 & 6.88 & 2.87 \\
\hline $\mathrm{PO}_{4}(\mu \mathrm{M}) / \mathrm{L}$ & nil & 23.04 & 1.68 & 0.00 & 3.98 & 1.07 & 0.00 & 2.50 & 0.64 & 0.00 & 1.92 & 0.81 \\
\hline $\mathrm{SiO}_{4}(\mu \mathrm{M}) / \mathrm{L}$ & nil & 105.74 & 19.91 & 1.88 & 83.11 & 17.17 & 0.11 & 3.08 & 1.31 & 0.23 & 1.20 & 0.51 \\
\hline OOM (mg/L) & nil & 50.60 & 4.11 & 0.24 & 116.00 & 21.68 & 0.16 & 4.60 & 1.41 & 0.16 & 6.44 & 1.78 \\
\hline
\end{tabular}

A total of 176 taxa of phytoplankton were identified in Naubaria site during the study period (Table 2). About $53.41 \%$ of them were either fresh or brackish water forms. Bacillariophyceae was the dominant group (Fig.2), forming numerically $95.11 \%$ of the main bulk and the most diversified group (39 genera, 113 taxa) but Dinophyceae showed lower numbers (10 genera, 16 species). The freshwater Chlorophyceae, Cyanophyceae and Euglenophyceae were represented by 22, 15 and 8 species,respectively, whereas silcoflagellates were represented by only two species (Table 3 ). The surface layer was more diversified (147 taxa) than the near bottom one (100 taxa). The majority of the recorded taxa (168) showed seasonal occurrence. However, four diatoms, two euglenophytes, one cyanophyte and one chlorophyte were perennial during the study period, among which were Gyrosigma attenuatum, Nitszchia palea, Nitzschia pungens and Nitzschia sigma, Euglena acus, euglena Eherenbergii, Oscillatoria brevis and Ankistrodesmus falcatus. High similarity of phytoplankton community composition between stations I \& II and between III\&IV was observed (Table 4). 
Table (2): A check list of the recorded species at the study area (west Alexandria) during March 2005-January 2006.

\begin{tabular}{|c|c|c|c|c|}
\hline Classes and species & $\begin{array}{c}\text { Naubaria } \\
\text { site }\end{array}$ & $\begin{array}{c}\text { El- } \\
\text { Mohandessin site }\end{array}$ & Marakia site & Marabella site \\
\hline \multicolumn{5}{|l|}{ Cyanophyceae } \\
\hline Anabaena spiroiides klebs & + & - & - & + \\
\hline Anabaena sp. & - & + & - & - \\
\hline Meismopedia punctata Meyen & + & - & - & - \\
\hline Oscillatoria aghardii Gomont & + & - & - & - \\
\hline Oscillatoria bervis Kütz & + & + & - & + \\
\hline Oscillatoria formosa Bory & + & - & - & - \\
\hline Oscillatoria limnetica Lemm. & + & + & - & - \\
\hline Oscillatoria limosa Agardh. & + & - & - & - \\
\hline Oscillatoria principes Vauch. & - & - & + & - \\
\hline Oscillatoria tenuis Agardh. & + & - & - & - \\
\hline Oscillatoria sp. & + & - & - & - \\
\hline Phormidium tenue (Menegh.) & + & - & - & - \\
\hline Phormidium sp. & + & - & - & - \\
\hline Spirulina major Kütz & + & - & - & - \\
\hline Spirulina okensis (C. Meyer) Geitler & + & - & - & - \\
\hline Spirulina platensis (Nordst) Geitler & + & - & + & - \\
\hline Spirulina subtillissina Kütz & + & - & + & + \\
\hline \multicolumn{5}{|l|}{ Euglenophyceae } \\
\hline Euglena acus Ehrenberg & + & + & + & + \\
\hline Euglena caudata Hübner & + & + & + & - \\
\hline Euglena ehrenbergii Ehrenberg & + & + & + & + \\
\hline Euglena gracilis Klebs & + & + & + & - \\
\hline Euglena granulata Klebs & - & + & - & - \\
\hline Euglena pisciformis Klebs & + & + & + & - \\
\hline Euglena proxima Dang. & + & - & - & - \\
\hline Euglena sp. & + & - & + & - \\
\hline Phacus caudatus Hübner & + & + & - & - \\
\hline \multicolumn{5}{|l|}{ Chlorophyceae } \\
\hline \multicolumn{5}{|l|}{ Ankistrodesmus falcatus Ralfs } \\
\hline Carteria sp. & + & + & + & + \\
\hline Chlamydomonas atactogama Korsch & + & - & - & - \\
\hline Chlamydomonas snowii Snow & + & - & - & - \\
\hline Chlamydomonas sp. & + & + & - & - \\
\hline Chlorella vulgaris Beij & + & + & + & + \\
\hline Chlorella sp. & + & - & - & - \\
\hline Clorogonium elegans Playt & - & - & + & - \\
\hline Closterium ehrenbergii Meneghini & + & - & - & - \\
\hline Closterium sp. & + & - & - & - \\
\hline Coelastrum microsporum Näg. & + & - & - & + \\
\hline Cosmarium reniforme (Rals.) & + & - & - & - \\
\hline Crucigenia tetrapida Kirchner & + & - & - & - \\
\hline Kirschneriella lunaris Krichner & + & - & - & - \\
\hline Kirschneriella obesa W. West & + & - & - & + \\
\hline
\end{tabular}




\section{Changes in phytoplankton community structure at three touristic sites at western Alexandria Beach}

Table (2): continued

\begin{tabular}{|c|c|c|c|c|}
\hline Classes & \begin{tabular}{|} 
Na \\
pria \\
site
\end{tabular} & $\begin{array}{c}\text { El- } \\
\text { Mohandessin } \\
\text { site }\end{array}$ & $\begin{array}{c}\text { Marakia } \\
\text { site }\end{array}$ & $\begin{array}{c}\text { Marabella } \\
\text { site }\end{array}$ \\
\hline Oocystis solitaria Wittr & + & + & - & - \\
\hline Planktosphaeria gelatinosa G.M. Smith & + & - & - & - \\
\hline Pediastrum clathratum Lemm. & + & - & - & - \\
\hline Pediastrum simplex Bailey & + & - & - & - \\
\hline Pediastrum tetras Ralfs & + & - & - & - \\
\hline Scendesmus bijugatus Kütz & + & - & - & - \\
\hline Scenedesmus dimorphus Tarp. & + & - & - & - \\
\hline Scenedesmus quadricauda Brébisson & + & + & - & - \\
\hline \multicolumn{5}{|l|}{ Bacillariophyceae } \\
\hline Acnanthes brevipes Agardh. & + & - & - & - \\
\hline Acnanthes longipes Agardh. & + & - & - & - \\
\hline Amphipora paludosa W. smith & + & - & + & - \\
\hline Amphora coffeaformis Agardh & + & + & - & - \\
\hline Amphora cymbifera Greg. & + & + & + & - \\
\hline Amphora decussata Grunow & + & - & - & - \\
\hline Amphora hyaline Kütz & - & - & + & - \\
\hline Amphora marina Smith & + & - & + & - \\
\hline Amphora proteus Greg. & + & - & - & - \\
\hline Amphora sp. & + & - & - & + \\
\hline Asterionella japonica Cleve & + & + & + & + \\
\hline Bacillaria paradoxa (Gmelin) Grunow & + & + & - & - \\
\hline Bacteriastrum delicatulum Cleve & + & - & - & - \\
\hline Bacteriastrum varians Lauder & - & - & + & - \\
\hline Biddulphia auriata Brébisson and Godey & + & + & - & - \\
\hline Biddulphia longicruris Greville & + & + & - & - \\
\hline Biddulphia mobiliensis Bailey & + & + & + & - \\
\hline Biddulphia rhombus (Ehrenberg)W. Smith & - & + & - & - \\
\hline Biddulphia sp. & - & + & - & - \\
\hline Campylostylus striatus Shalbolt & + & - & - & - \\
\hline Cerataulina bergonii $\mathrm{H}$. Peragallo & + & - & - & - \\
\hline Chaetoceros affinis Lauder & + & + & + & - \\
\hline Chaetoceros brevis Schütt & - & - & + & - \\
\hline Chaetoceros compressus Lauder & + & - & - & - \\
\hline Chaetoceros curvisetus Cleve & + & + & + & + \\
\hline Chaetoceros decipiens Cleve & - & - & - & + \\
\hline Chaetoceros gracilis Schütt & + & - & - & - \\
\hline Chaetoceros sp. & + & - & + & - \\
\hline Climacosphenia moniligera Ehrenberg & + & + & + & + \\
\hline Cocconeis placentula Ehrenberg & + & + & + & + \\
\hline Coscinodiscus centralis Ehrenberg & + & + & - & + \\
\hline Coscinodiscus excentricus Ehrenberg & + & + & + & - \\
\hline Coscinodiscus marginatus Ehrenberg & - & + & - & - \\
\hline Coscinodiscus radiatus Ehrenberg & + & + & - & - \\
\hline Cyclotella glomerata Thwaitwes & + & + & - & - \\
\hline Cyclotella kütziginiana Thwaitwes & + & + & + & + \\
\hline Cyclotella mengheniana Kütz & + & - & - & - \\
\hline Cyclotella operculata A..g. & + & - & - & - \\
\hline Cyclotella sp. & + & + & + & - \\
\hline Cymbella turgida Greg. & + & - & - & - \\
\hline Cymbella ventricosa Kütz & + & - & - & - \\
\hline Cymbella sp. & - & - & + & - \\
\hline
\end{tabular}


Table (2): continued

\begin{tabular}{|c|c|c|c|c|}
\hline Classes & \begin{tabular}{|}
$\mathrm{Na}$ \\
aria \\
site
\end{tabular} & $\begin{array}{c}\text { El- } \\
\text { Mohandessin } \\
\text { site }\end{array}$ & $\begin{array}{c}\text { Marakia } \\
\text { site }\end{array}$ & $\begin{array}{c}\text { Marabella } \\
\text { site }\end{array}$ \\
\hline Gomphonema acuminatum Kütz & + & - & - & - \\
\hline Gramatophora marina (Lyngb.) Kütz & + & - & + & + \\
\hline Gramatophora oceanica (Her.) Grunow & + & + & + & - \\
\hline Gramatophora sp. & - & - & - & + \\
\hline Gyrosigma acuminatum Kütz & + & - & - & + \\
\hline Gyrosigma attenuatum Kütz & + & + & + & - \\
\hline Gyrosigma sp. & + & - & + & + \\
\hline Hemiaulus hauckii Grunow & + & + & - & + \\
\hline Hemiaulus membranceus Cleve & + & - & - & - \\
\hline Hemiaulus sinensis Grevill & + & + & - & - \\
\hline Laudrea borealis Gran & + & - & - & - \\
\hline Leptocylindrus danicus Cleve & + & + & - & + \\
\hline Licmophora abbreviata Agardh. & + & + & + & + \\
\hline Licmophora gracilis Ehrenberg & + & + & + & + \\
\hline Licmophora sp. & + & - & + & + \\
\hline Mastogloia Smithii Thwaites & + & - & - & - \\
\hline Melosira granulata Ehrenberg & + & + & - & - \\
\hline Melosira moniliforms Müller (Agardh.) & + & + & + & - \\
\hline Melosira varians C.A.A. Agardh. & + & - & - & - \\
\hline Melosira Jurgensii Aghardh. & + & - & - & - \\
\hline Navicula bombus Ehrenberg & + & + & - & - \\
\hline Navicula carnifera Grunow & + & - & - & - \\
\hline Navicula crabo E. & + & - & - & - \\
\hline Navicula cryptocephala Kütz & + & + & + & + \\
\hline Navicula cuspidata Kütz & + & - & + & + \\
\hline Navicula dicephala Ehrenberg & + & + & + & - \\
\hline Navicula digito radiata Grég. & - & - & + & - \\
\hline Navicula didyma Ehrenberg & + & - & + & + \\
\hline Navicula directa Grég. & - & - & + & - \\
\hline Navicula fortis Grég. & + & - & - & - \\
\hline Navicula fusca Grég. & - & - & + & - \\
\hline Navicula gracilis Ehrenberg & + & + & + & - \\
\hline Navicula humersoa Brébisson & + & + & + & - \\
\hline Navicula littoralis Donk. & + & - & - & - \\
\hline Navicula marina Ralfs & + & - & - & - \\
\hline Navicula placentula Ehrenberg & + & - & - & - \\
\hline Navicula rhyncocephala Kütz & + & - & - & - \\
\hline Navicula viridula Kütz & + & - & + & + \\
\hline Navicula sp. & + & + & + & + \\
\hline Nitzschia acicularis W. Smith & + & + & + & - \\
\hline Nitzschia acuminata W. Smith & + & - & - & - \\
\hline Nitzschia closterium W. Smith & + & + & - & - \\
\hline Nitzschia commutata Grunow & + & - & - & - \\
\hline Nitzschia delicatissima Cleve & + & + & - & - \\
\hline Nitzschia kütziginiana Hilse & + & + & + & + \\
\hline Nitzschia lanceolata W. Smith & + & + & - & - \\
\hline Nitzschia linearis (Agardh.) Smith & + & - & - & - \\
\hline Nitzschia longissima (Brébisson) Ralfs & + & + & + & + \\
\hline Nitzschia microcephala Grunow & + & - & - & - \\
\hline Nitzschia obtusa W. Smith & + & - & - & - \\
\hline Nitzschia pacifica Cupp. & + & + & - & - \\
\hline Nitzschia palea Kütz & + & + & + & + \\
\hline
\end{tabular}




\section{Changes in phytoplankton community structure at three touristic sites at western Alexandria Beach}

Table (2): continued

\begin{tabular}{|c|c|c|c|c|}
\hline Classes & \begin{tabular}{|c} 
Na \\
hria \\
site
\end{tabular} & $\begin{array}{c}\text { El- } \\
\begin{array}{c}\text { Mohandessin } \\
\text { site }\end{array} \\
\end{array}$ & $\begin{array}{c}\text { Marakia } \\
\text { site }\end{array}$ & $\begin{array}{c}\text { Marabella } \\
\text { site }\end{array}$ \\
\hline $\begin{array}{l}\text { Nitzschia panduriformis var. peralbata } \\
\text { Peragallo }\end{array}$ & + & - & + & - \\
\hline Nitzschia panduriformis var. minor Gunow & + & - & - & - \\
\hline Nitzschia pungens Cleve & + & + & + & + \\
\hline Nitzschia sigma Kütz & + & + & + & + \\
\hline Nitzschia sp. & + & + & + & + \\
\hline Plagiogramma vanheurckii Grunow & + & + & - & - \\
\hline Pleurosigma acuminatum Grunow & + & - & - & - \\
\hline Pleurosigma elongatum W. Smith & - & + & - & - \\
\hline Pleurosigma rigidum W.Smith & + & - & - & - \\
\hline Pleurosigma sp. & + & + & - & - \\
\hline Rhizosolenia alata Brightwell & + & - & + & - \\
\hline Rhizosolenia bergonii H. Peragallo & + & + & + & - \\
\hline Rhizosolenia calcar - avis M. Sclultze & + & - & + & + \\
\hline Rhizosolenia delicatula Cleve & + & + & - & - \\
\hline Rhizosolenia fragellissima Bergon & + & + & + & - \\
\hline $\begin{array}{l}\text { Rhizososlenia hebetata (Bailey) Gran, } \\
\text { emend. }\end{array}$ & + & + & - & - \\
\hline Rhizosolenia setigera Brightwel & + & + & + & - \\
\hline Rhizosolenia stolterforthii $\mathrm{H}$. Peragallo & + & - & - & - \\
\hline Rhizosolenia styliformis Brightwell & + & - & - & - \\
\hline Rhizosolenia sp. & + & - & + & - \\
\hline $\begin{array}{l}\text { Shröderella delicatula (H. peragallo) } \\
\text { pavillard }\end{array}$ & + & - & - & - \\
\hline Skeletonema costatum (Greville) cleve & + & + & + & - \\
\hline Stauroneis quarnerensis Grunow & - & + & - & - \\
\hline Streptotheca thamensis Shrubsole & - & + & - & - \\
\hline Striatella delicatula (Kütz) Grunow & + & - & + & - \\
\hline Striatella unipunctata Lyngb & + & - & - & + \\
\hline Surirella factuosa Grunow & - & + & - & - \\
\hline Surirella ovata Kütz & + & - & - & - \\
\hline Surirella pandura Peragallo & + & - & - & - \\
\hline Surirella sp. & + & - & - & - \\
\hline Synedra acus Kütz & + & - & - & - \\
\hline Synedra affinis Kütz & + & + & - & - \\
\hline Synedra ulna (Nitzsch) & + & + & + & + \\
\hline $\begin{array}{l}\text { Tabellaria fenestrata var. intermedia } \\
\text { Grunow }\end{array}$ & - & - & + & + \\
\hline Thalassionema nitzschoïdes Gunow & + & - & + & + \\
\hline Thalassiosira rotula Meunier & + & - & - & - \\
\hline Thalassiothrix frauenfeldii Grunow & + & + & + & + \\
\hline \multicolumn{5}{|l|}{ Dinophyceae } \\
\hline Amphidinum sp. & + & - & - & - \\
\hline Creatium furca Ehrenberg & + & - & + & + \\
\hline Ceratium fusus Ehrenberg & + & - & + & - \\
\hline Ceratium pentagonum Gourret & - & - & + & - \\
\hline Dinophysis caudata Savielle-Kent & + & + & - & - \\
\hline Dinophysis tripos Gourret & - & - & + & - \\
\hline Dinophysis sp. & - & - & + & - \\
\hline Diplopsalis rotunda Lebour (Wood) & + & + & - & + \\
\hline Gonyaulax polyedra Stein & - & - & - & + \\
\hline Gonyaulax polygramma Stein & + & + & + & - \\
\hline Gongaulax sp. & - & - & + & - \\
\hline
\end{tabular}


Table (2): continued

\begin{tabular}{|l|c|c|c|c|}
\hline Classes & $\begin{array}{c}\text { Na } \\
\text { ria } \\
\text { site }\end{array}$ & $\begin{array}{c}\text { El- } \\
\text { Mohandessin } \\
\text { site }\end{array}$ & $\begin{array}{c}\text { Marakia } \\
\text { site }\end{array}$ & $\begin{array}{c}\text { Marabella } \\
\text { site }\end{array}$ \\
\hline Gtmnodinium rotundatum Klebs & + & - & - & - \\
\hline Gymnodinium sp. & + & - & - & + \\
\hline Oxytoxum sceptrum Stein (Schröder) & + & + & + & - \\
\hline Oxytoxum sp. & - & + & - & - \\
\hline Prorocentrum micans Ehrenberg & + & + & - & - \\
\hline Prorocentrum sp. & + & + & + & + \\
\hline Protoperidinium cerasus Paulsen & + & + & - & - \\
\hline Protopenidinium conicoïdes Paulsen & + & - & + & + \\
\hline $\begin{array}{l}\text { Protoperidinium conicum } \text { Ostenfield and } \\
\text { Schmidt }\end{array}$ & + & + & - & - \\
\hline Protopenidinium depressum Bailey & + & + & + & + \\
\hline Protopenidinium sp. & + & + & - & + \\
\hline Pyrocystis sp. & - & - & & \\
\hline Silicoflagellates & & + & + & - \\
\hline Dictyocha fibula Ehrenberg & + & + & + & + \\
\hline Ebria sp. & + & + & & \\
\hline
\end{tabular}




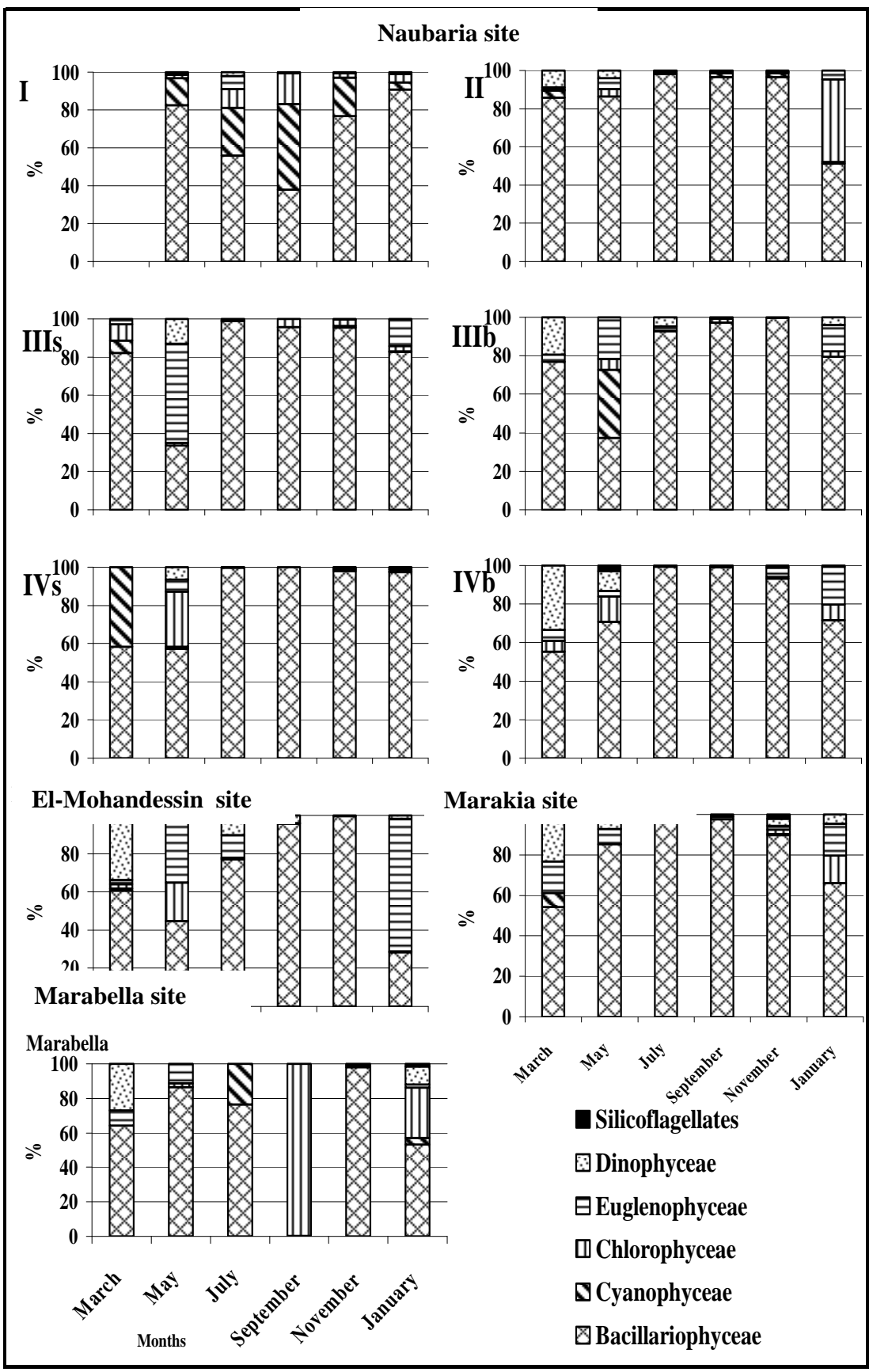

Fig. (2): The relative abundance of the different groups of phytoplankton at Naubaria site, El-Mohandessin, Marakia and Marabella sites during March 2005-January 2006. 
The frequency of appearance of the different species revealed that 41 taxa were observed throughout the Naubaria site, 18 taxa were observed at the three stations, 39 taxa at two stations and 75 taxa were found in only one station, regardless of great differences in their densities among stations. The total number of species demonstrated more pronounced variations on the temporal scale than the spatial one (Fig. 3). It varied between 42 taxa in March and 91 taxa in September, while on the spatial scale varied between 72 taxa at stations III and IV and 87 taxa at station II. The high diversity at the latter station was attributed to the higher number of freshwater forms (58.6\% of the total number) (Fig. 4). The freshwater forms were mostly restricted to low salinity (7.67 12.35 PSU) with the presence of the genera Pediastrum, Oscillatoria, Euglena, Cyclotella, and Synedra (Table 5).

Indices of diversity, evenness and species richness were employed as parameters to define the structure of phytoplankton community in Naubaria site. Generally, the Shanon-Weaver diversity index at the surface water showed high values at station I (average 2.48 nats) and decreased towards the sea direction to reach minimum average of 1.38 nats at station IV. The high diversity was attributed to the presence of fresh, brackish, and marine phytoplankton species. The diversity index ranged between 0.53 nats (St. IV, November) and 2.99 nats (St.I, November) (Fig. 5). The evenness index showed the same distribution pattern, and fluctuated between 0.12 (St. IV, November) and 0.70 (St. IV, May) (Fig. 3). For the near bottom layer, the highest values were 2.8 nats for ShanonWeaver diversity indices and 0.81 for evenness indices (St.IV, May), accompanied with 73 species. The lowest values were 0.14 nats and 0.04 (St.III, November), for diversity and evenness, respectively, accompanied with low number of species and high total count. The richness index ranged between 1.42 (St.II, July) and 4.46 (St.II, November) for the surface water and between 1.14 (St.IV, July) and 3.77 (ST. III, September) for near bottom layer (Fig. 3). The low diversity index indicates a weak community structure point to the dominance of one species recorded in November at the surface water of St. IV and near bottom layer at St. III, both due to the bloom of Skeletonema costatum, which formed $91.4 \% \& 98 \%$ to the total phytoplankton count, respectively.

The phytoplankton abundance was generally low at Naubaria site, attaining at surface layer an average of $46 \times 10^{3}$ unit/L, whereas it decreased near the bottom layer to $23 \times 10^{3}$ unit/L. 
Table (3): Number of genera and taxa of different groups, total average $\left(\mathrm{u} / \mathrm{L} \times 10^{3}\right)$ and relative abundance at the study area during March 2005 - January 2006.

\begin{tabular}{|c|c|c|c|c|c|c|c|c|c|c|c|c|c|c|c|c|}
\hline \multirow[b]{2}{*}{ Group } & \multicolumn{4}{|c|}{ Naubaria site } & \multicolumn{3}{|c|}{ El-Mohandessin site } & \multirow[b]{2}{*}{ 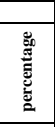 } & \multicolumn{4}{|c|}{ Marakia site } & \multicolumn{4}{|c|}{ Marabella site } \\
\hline & 芯 & 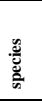 & 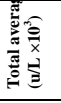 & 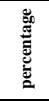 & 迆 & 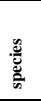 & 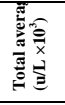 & & 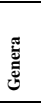 & $\begin{array}{l}\stackrel{\mathscr{\nu}}{u} \\
\text { के } \\
\text { के }\end{array}$ & 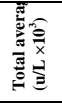 & 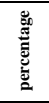 & 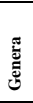 & $\begin{array}{l}\mathscr{\Xi} \\
\text { के } \\
\text { के }\end{array}$ & 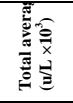 & 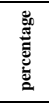 \\
\hline Bacillariophyceae & 40 & 113 & 36.770 & 95.11 & 25 & 61 & 137.322 & 96.94 & 24 & 56 & 2.407 & 91.31 & 20 & 3 & 6.134 & 1.341 \\
\hline Cyanophyceae & 5 & 15 & 0.731 & 1.89 & 2 & 3 & 0.037 & 0.02 & 2 & 3 & 0.013 & 0.49 & 3 & 3 & 0.007 & 0.001 \\
\hline Chlorophyceae & 14 & 22 & 0.669 & 1.73 & 6 & 6 & 0.104 & 0.07 & 4 & 4 & 0.037 & 1.4 & 5 & 5 & 450.992 & 98.62 \\
\hline Euglenophyceae & 2 & 8 & 0.281 & 0.73 & 2 & 7 & 0.308 & 2.17 & 1 & 6 & 0.084 & 3.19 & 1 & 2 & 0.048 & 0.011 \\
\hline Dinophyceae & 10 & 16 & 0.202 & 0.52 & 6 & 11 & 1.097 & 0.78 & 6 & 12 & 0.084 & 3.19 & 7 & s & 0.117 & 0.026 \\
\hline Silicoflagellates & 2 & 2 & 0.008 & 0.02 & 2 & 2 & 0.023 & 0.02 & 2 & 2 & 0.011 & 0.42 & 1 & & 0.003 & 0.001 \\
\hline Total & 73 & 176 & 38.661 & 100 & 43 & 90 & 141.663 & 100 & 39 & 83 & 2.636 & 100 & 37 & 5 & 457.301 & 100 \\
\hline
\end{tabular}

Table (4): Number of species of different groups at the sampled stations of Naubaria site during March 2005 - January 2006.

\begin{tabular}{|c|c|c|c|c|c|c|c|}
\hline \multirow{2}{*}{ Groups } & \multirow{2}{*}{ Stations } & \multirow{2}{*}{ I } & \multirow{2}{*}{ II } & \multicolumn{2}{|c|}{ III } & \multicolumn{2}{|c|}{ IV } \\
\hline & & & & $\mathrm{S}$ & B & $\mathrm{S}$ & B \\
\hline Bacillariophyceae & & 57 & 60 & 46 & 50 & 48 & 59 \\
\hline Cyanophyceae & & 12 & 6 & 5 & 3 & 6 & 0 \\
\hline Chlorophyceae & & 6 & 7 & 8 & 6 & 7 & 5 \\
\hline Euglenophyceae & & 6 & 5 & 5 & 5 & 3 & 5 \\
\hline Dinophyceae & & 3 & 7 & 8 & 10 & 7 & 6 \\
\hline Dictyophyceae & & 0 & 2 & 0 & 1 & 1 & 1 \\
\hline Total & & 84 & 87 & 72 & 75 & 72 & 76 \\
\hline
\end{tabular}




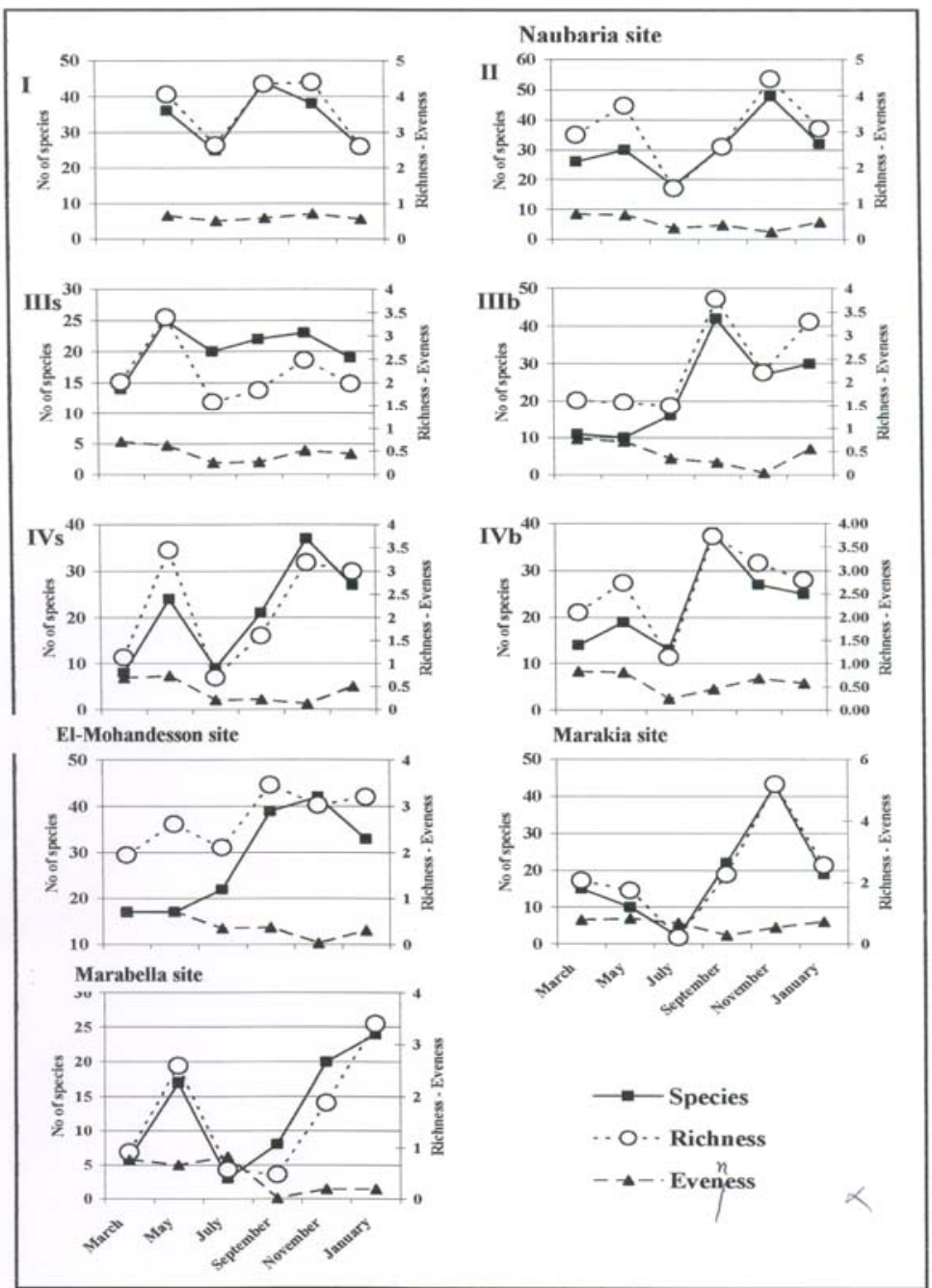

Fig. (3): The bimonthly variations in species number, richness and evenness at Naubaria site, El-Mohandessin, Marakia and Marabella sites during March 2005 January 2006. 


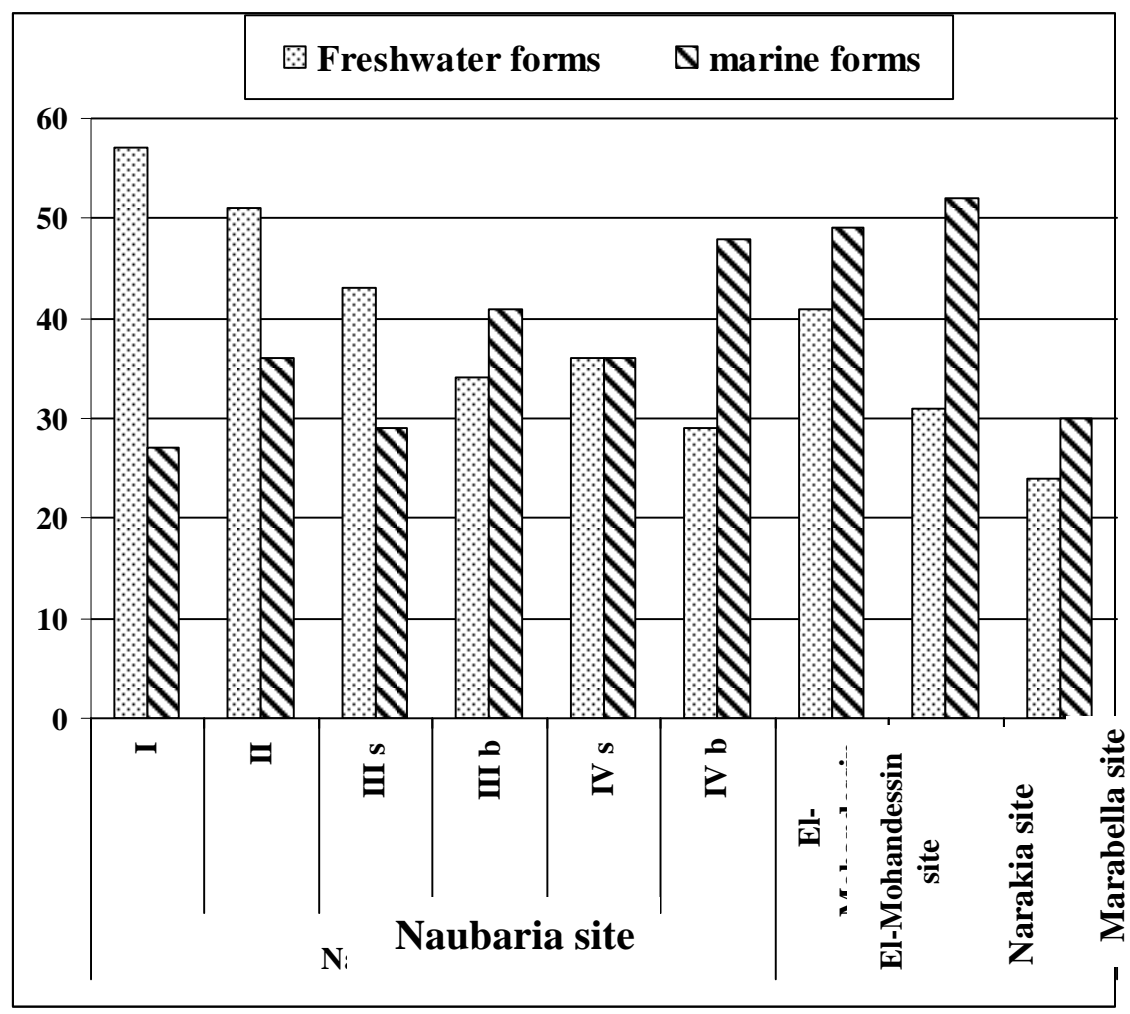

Fig.(4): The number of freshwater and marine plytoplankton at Naubaria site, El-Mohandessin, Marakia and Marabella sites during Marh 2005- January 2006. 
Table (5): Dominant phytoplankton species ( $>1 \%$ of total count), salinity range and salinity at maximum existence at Naubaria site during March 2005 - January2006.

\begin{tabular}{|c|c|c|c|c|c|c|c|c|}
\hline \multirow[t]{2}{*}{ Stations } & \multirow{2}{*}{ I } & \multirow{2}{*}{ II } & \multicolumn{2}{|c|}{ III } & \multicolumn{2}{|c|}{ IV } & \multirow{2}{*}{ S\% range } & \multirow{2}{*}{$\begin{array}{c}\mathrm{S} \% \\
\text { maximum }\end{array}$} \\
\hline & & & $\mathbf{S}$ & B & $\mathbf{S}$ & B & & \\
\hline \multicolumn{9}{|l|}{ Cyanophyceae } \\
\hline Merismopedia punctata & 0 & 2.1 & 0 & 0 & 0 & 0 & $21.8-38.5$ & 28.7 \\
\hline Ocillatoria aghardhii & 1.04 & 0 & 0 & 0 & 0 & 0 & $7.6-12.35$ & 10.15 \\
\hline Oscillatoria bervis & 16.5 & 0 & 0 & 0 & 0 & 0 & $7.6-12.35$ & 9.55 \\
\hline Oscillatoria limosa & 4.3 & 0 & 0 & 0 & 0 & 0 & $7.6-12.35$ & 9.55 \\
\hline Oscillatoria tenuis & 1.3 & 0 & 0 & 0 & 0 & 0 & $7.6-12.35$ & 8.92 \\
\hline Total Cyanophyceae & 23.14 & 2.1 & $\mathbf{0}$ & $\mathbf{0}$ & $\mathbf{0}$ & $\mathbf{0}$ & & \\
\hline \multicolumn{9}{|l|}{ Euglenophyceae } \\
\hline Euglena acus & 0 & 0 & 0 & 0 & 0 & 1.7 & $39.09-39.72$ & 39.13 \\
\hline \multicolumn{9}{|l|}{ Chlorophyceae } \\
\hline Chlamydomonas atactogama & 0 & 0 & 1.3 & 0 & 0 & 0 & $37.64-39.60$ & 39.6 \\
\hline Chlorella vulgaris & 1.1 & 0 & 0 & 0 & 0 & 0 & 7.6-12.35 & 12.3 \\
\hline Chlorella sp. & 0 & 2.9 & 0 & 0 & 0 & 0 & $21.8-38.5$ & 21.8 \\
\hline Pediastrum simplex & 7.7 & 0 & 0 & 0 & 0 & 0 & $7.6-12.35$ & 9.55 \\
\hline Total Chlorophyceae & 8.8 & 2.9 & 1.3 & $\mathbf{0}$ & $\mathbf{0}$ & $\mathbf{0}$ & & \\
\hline \multicolumn{9}{|l|}{ Bacillariophyceae } \\
\hline Amphiprora paludosa & 2.5 & 0 & 0 & 0 & 0 & 0 & $7.6-12.35$ & 12.35 \\
\hline Bacillaria paradoxa & 1.2 & 0 & 0 & 0 & 0 & 0 & $7.6-12.35$ & 9.55 \\
\hline Chaetoceros affinis & 0 & 0 & 0 & 0 & 1.4 & 0 & $37.37-39.72$ & 39.33 \\
\hline Chaetoceros curvisetus & 0 & 0 & 0 & 0 & 2.2 & 0 & $37.37-39.72$ & 39.09 \\
\hline Cyclotella glomerata & 0 & 2.2 & 0 & 1.4 & 0 & 0 & $21.79-39.33$ & 35.2 \\
\hline Cyclotella Kütziginiana & 1.3 & 30.5 & 34.4 & 8.7 & 22.2 & 43.2 & 7.6-39.7 & 37.37 \\
\hline Gyrosigma attenuatum & 1.1 & 0 & 0 & 0 & 0 & 0 & $7.6-12.35$ & 9.55 \\
\hline Melosira moniliforms & 10.3 & 0 & 0 & 0 & 0 & 0 & $7.6-12.35$ & 8.92 \\
\hline Navicula cryptocephala & 14.8 & 1.9 & 0 & 0 & 0 & 0 & $7.6-38.50$ & 12.35 \\
\hline Navicula dicephala & 2.6 & 0 & 0 & 0 & 0 & 0 & $7.6-12.35$ & 12.35 \\
\hline Navicula gracilis & 1.4 & 0 & 0 & 0 & 0 & 0 & $7.6-12.35$ & 12.35 \\
\hline Navicula sp. & 3.5 & 0 & 0 & 0 & 0 & 0 & 7.6-12.35 & 12.35 \\
\hline Nitzschia closterium & 2 & 0 & 0 & 0 & 0 & 0 & $7.6-12.35$ & 12.35 \\
\hline Nitzschia longissima & 2.2 & 0 & 0 & 0 & 0 & 0 & $7.6-12.35$ & 9.55 \\
\hline Nitzschia pacifica & 0 & 0 & 1.2 & 0 & 0 & 0 & $37.64-39.65$ & 37.64 \\
\hline Nitzschia palea & 3.5 & 0 & 0 & 0 & 0 & 0 & $7.6-12.35$ & 9.55 \\
\hline Nitzschia panduriform var.minor & 0 & 0 & 20.9 & 0 & 0 & 0 & $37.64-39.65$ & 39.6 \\
\hline Nitzschia pungens & 1.0 & 2.7 & 0 & 2.7 & 3.8 & 4.7 & $7.6-39.72$ & 39.13 \\
\hline Nitzschia sigma & 4.2 & 2.7 & 1.7 & 1.9 & 1.9 & 2.6 & $7.6-39.72$ & 10.15 \\
\hline Nitzschia $\mathrm{sp}$. & 1.3 & 0 & 0 & 0 & 0 & 0 & $7.6-12.35$ & 12.35 \\
\hline Rhizosolenia fragellissima & 0 & 17.96 & 22.2 & 1.8 & 5.5 & 13.0 & $21.79-39.72$ & 37.64 \\
\hline Skeletonema costatum & 0 & 9.8 & 0 & 59.4 & 16.6 & 0 & $21.79-39.33$ & 39.32 \\
\hline Synedra ulna & 2.9 & 0 & 0 & 0 & 0 & 0 & $7.6-12.35$ & 9.55 \\
\hline Total Bacillariophyceae & 55.8 & 86.16 & 91.5 & 94.5 & 98.7 & 79.9 & & \\
\hline
\end{tabular}

However, phytoplankton abundance displayed great variations. On the spatial scale, phytoplankton abundance at surface layer increased towards the sea sustaining the lowest count at station I $\left(11 \times 10^{3}\right.$ unit/L), whereas the highest count $\left(74 \times 10^{3}\right.$ unit/L) was recorded at station IV. On the other hand, phytoplankton abundance at near bottom layer was $35 \times 10^{3}$ unit/L and $11 \times 10^{3}$ unit/L (St.III\&IV), respectively. Bimonthly variations at the surface layer showed two distinguished peaks and a minor one. The first peak occurred during July at stations II-IV $\left(113 \times 10^{3}-162 \times 10^{3}\right.$ unit/L $)$. Cyclotella Kütziginiana was the leader species (58 - 86\% to the total count). The second peak occurred in September at station IV $\left(244 \times 10^{3}\right.$ unit/L), resulting from the flourishing of the diatom Rhizosolenia setigera which formed $82.4 \%$ of the total count. A minor peak was recorded in November at station IV $\left(79 \times 10^{3}\right)$, resulting from Skeletonema costatum, which formed collectively $91.42 \%$ of the total count. 


\section{Changes in phytoplankton community structure at three touristic sites at western Alexandria Beach}

On the other hand, the temporal and spatial distribution near the bottom layer demonstrated similar patterns to that at surface layer and recorded the same dominant species but with different percentages (Fig.5).

Total chlorophyll at surface layer ranged from 0.46 (St.IV, July) to $15.42 \mu \mathrm{g} / \mathrm{L}$ (St.III, July) (Fig.6), showing slight increase at near the bottom layer. Spatially, station II recorded the highest concentration average $(4.01 \mu \mathrm{g} / \mathrm{L})$. On the other hand, bimonthly variations showed a peak of chlorophyll- $a$ during July $(7.73 \mu \mathrm{g} / \mathrm{L})$, coinciding with the maximum abundance of the two species Cyclotella Kütziginiana and Rhizosolenia fragellissima. In general, total chlorophyll at near bottom layer followed the same pattern, recording the highest concentration during July $(13.57 \mu \mathrm{g} / \mathrm{L})$. However, the net chlorophyll $<$ 20 was higher in concentration than chlorophyll $>20$ at both layers (Fig. 6).

\section{EL-Mohandessin touristic village site:}

Water temperature attained the classical seasonal variations of the water at the Egyptian Mediterranean coast $\left(15.34-30.00^{\circ} \mathrm{C}\right)$. The water salinity was slightly lower than the expected values in the Egyptian coastal waters (36.88$38.80 \mathrm{PSU})$. Water transparency fluctuated between 100 and $150 \mathrm{~cm}$. pH values lie on the alkaline side (8.08-8.24). The dissolved oxygen concentration ranged between 4.01 and $13.57 \mathrm{mg} \mathrm{O}_{2} / \mathrm{L}$. Ammonia ranged from 1.80 to $4.72 \mu \mathrm{M} / \mathrm{L}$; nitrite from 0.00 to $1.70 \mu \mathrm{M} / \mathrm{L}$; nitrate from 0.00 to $28.93 \mu \mathrm{M} / \mathrm{L}$; phosphate from 0.00 to $3.98 \mu \mathrm{M} / \mathrm{L}$ and silicate from 1.88 to $83.11 \mu \mathrm{M} / \mathrm{L}$. The oxidizable organic matter fluctuated between 0.24 (March) to $11.96 \mathrm{mg} / \mathrm{L}$ (January) with an abnormal value of $116 \mathrm{mg} / \mathrm{L}$ recorded in (May) (Table 1).

The phytoplankton community in water samples taken at ElMohandessin village comprised a total of 90 taxa (Table 2), of which 49 taxa were purely marine and 41 were either fresh or brackish water forms (Fig. 4). Six algal groups were represented, namely Bacillariophyceae (25 genera, 61 taxa), Dinophyceae (6 genera, 11 species), Chlorophyceae (6 genera, 6 species), Euglenophyceae ( 2 genera, 7 species), Cyanophyceae ( 2 genera, 3 species), and silicoflagellates ( 2 genera, 2 species) (Table 3 ). The phytoplankton diversity displayed bimonthly variations, where the number of species varied between 17 taxa in March, May and 42 taxa in November (Fig. 3). Bacillariophyceae was the dominant group forming numerically $96.94 \%$ of the total count generally (Table 3). However, the Bacillariophyceae predominated in March, May, July, September, attaining $99.81 \%$ in November, the lowest percentage $(44.81 \%)$ was recorded in May. In contrast, euglenophytes was dominant in January, forming $69.34 \%$ of the total count due to the absolute dominance of Euglena acus (Fig. 2). 


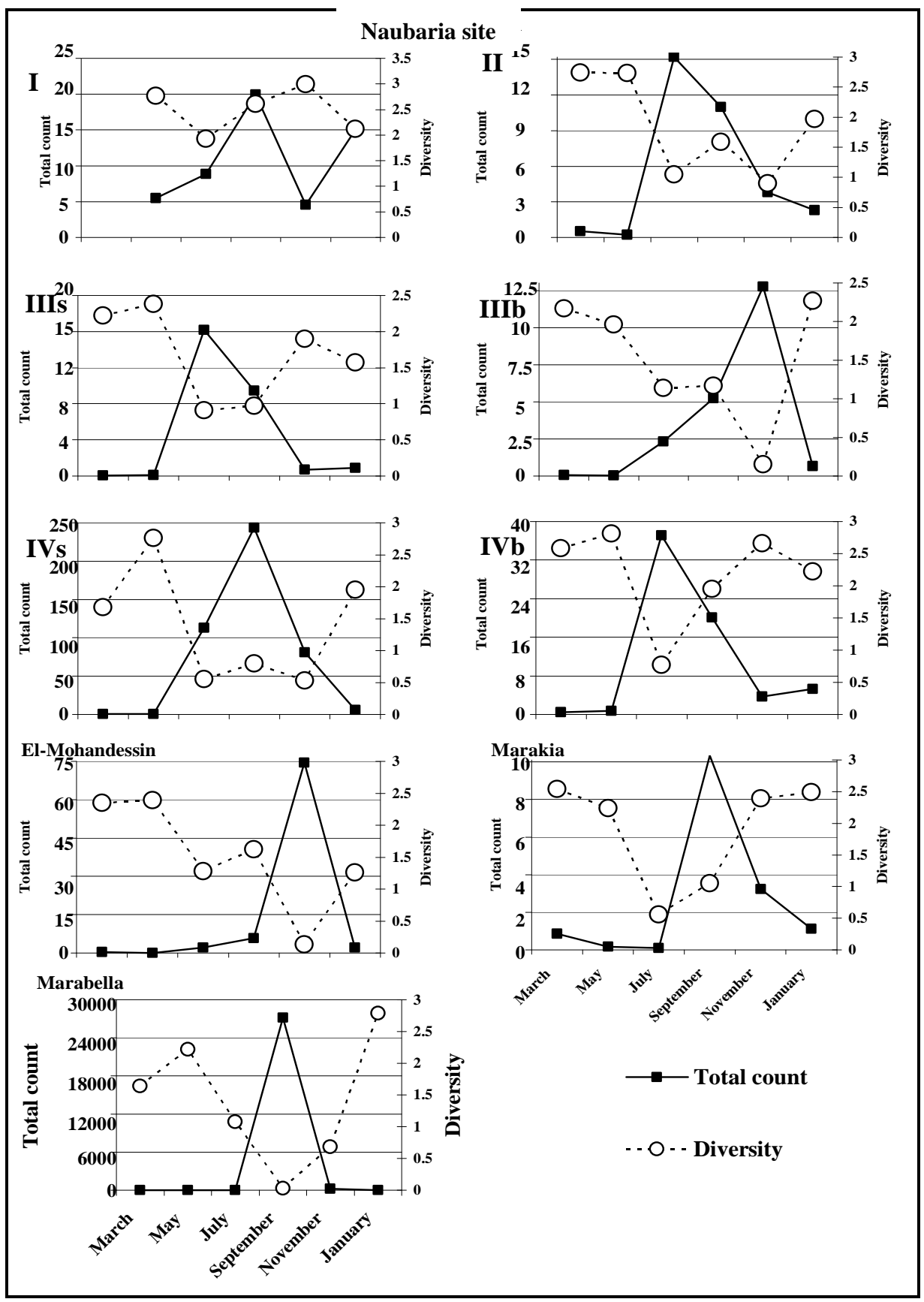

Fig. (5): The phytoplankton count $\left(\mathrm{u} / \mathrm{L} \times 10^{3}\right)$ and diversity (nats) index at Naubaria site, El-Mohandessin, Marakia and Marabella sites during March 2005 - January 2006. 


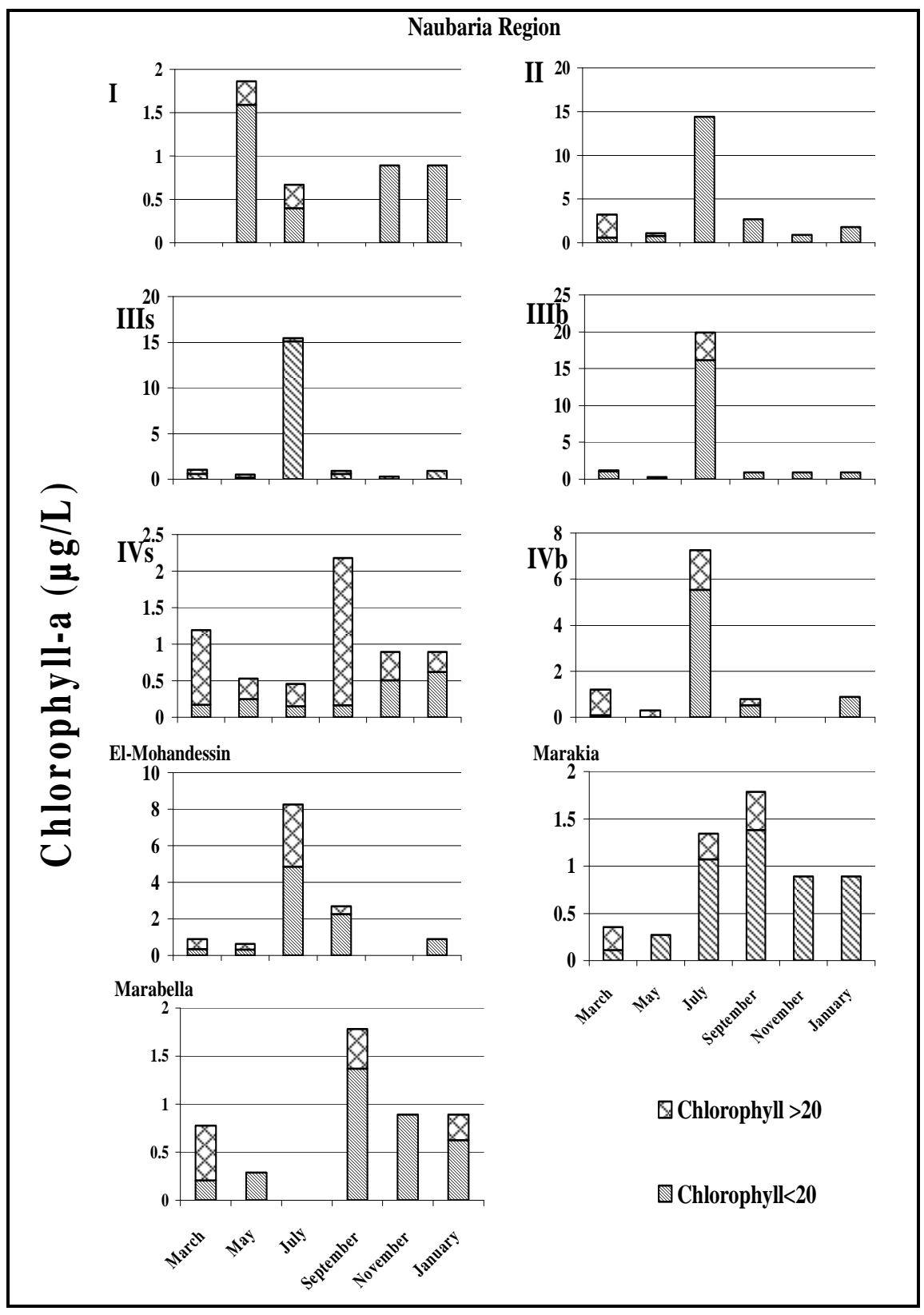

Fig. (6): The total chlorophyll-a ( $\mu \mathrm{g} / \mathrm{L})$, chlorophyll $>20$ and $<20$ at Naubaria site, ElMohandessin, Marakia and Marabella sites during March 2005 - January 2006. 
The absolute values of diversity index fluctuated from a minimum value (0.13 nats) in November to a maximum value (2.39 nats) in May (Fig. 5), antagonizing with total phytoplankton abundance. The evenness index showed the same distribution pattern, ranging from 0.03 in November to 0.71 in May. On the other hand, the richness values displayed narrow temporal variation from 1.95 in March to 3.46 in September (Fig. 3).

During the study period, phytoplankton abundance at El-Mohandessin village attained a total average of $142 \times 10^{3}$ unit/liter (Table 3), whereas bimonthly variations showed one distinguished peak in November, reaching a maximum value of $745 \times 10^{3}$ unit/liter. In contrast, the minimum value (453unit/liter) was recorded in May (Fig. 5). However, the flourishing of the diatom Skeletonema costatum was responsible for the peak, contributing $98.49 \%$ of the total count.

Generally, the concentration of total chlorophyll- $a$ was low, ranging from $0.46 \mu \mathrm{g} / \mathrm{L}$ in May to $8.26 \mu \mathrm{g} / \mathrm{L}$ in July. On the other hand, the net chlorophyll contributed to the total chlorophyll by a higher percentage $(72.85 \%)$ than the chlorophyll $>20$ (Fig. 6).

\section{Marakia touristic village site:}

At this site, water temperature ranged from 15.56 to $30.00^{\circ} \mathrm{C}$. Water salinity was slightly higher than the normal values of the Sea (38.65-39.80PSU). Water transparency varied from 100 to $200 \mathrm{~cm}$. $\mathrm{pH}$ values lied on the alkaline side, showing a very narrow range (8.03-8.09). Dissolved oxygen concentration ranged between 5.19 and $13.57 \mathrm{mg} \mathrm{O} / \mathrm{L}$. The nutrient salts and oxidizable organic matter were at low levels during the study period, such that, ammonia concentration was between 0.14 and $24.25 \mu \mathrm{M} / \mathrm{L}$, nitrite concentration was between 0.00 and $0.30 \mu \mathrm{M} / \mathrm{L}$, nitrate concentration was between 0.10 and 4.01 $\mu \mathrm{M} / \mathrm{L}$, phosphate concentration was between 0.00 and $2.50 \mu \mathrm{M} / \mathrm{L}$, silicate concentration between 0.11 and $3.08 \mu \mathrm{M} / \mathrm{L}$ and oxidizable organic matter represented as $\mathrm{O}_{2}$ was between 0.16 and $4.60 \mathrm{mg} / 1$ (Table 1).

The phytoplankton community in the water samples collected at Marakia village site comprised a total of 83 taxa (Table 2), of which the majority were typical marine forms (52 taxa), whereas 31 taxa were either brackish or freshwater forms (Fig. 4). The Bacillariophyceae was the most diversified group (20 genera, 56 taxa) followed by Dinophyceae (6 genera, 12 species), Euglenophyceae (1genus, 6 species), Chlorophyceae (4 genera, 4 species), Cyanophyceae (2 genera, 3 species) and Silicoflagellates (2genera, 2 species) (Table 3). Bacillariophyceae was the dominant group forming numerically $91.31 \%$ of algal community (Table 3). However, Bacillariophyceae predominated during the study period with the lowest percentage $(54.22 \%)$ recorded during March (Fig. 2). The number of phytoplankton species displayed obvious bimonthly variations, where only two purely marine forms were recorded in July, whereas the highest number (43 taxa) was recorded in November comprising 51.16\% freshwater forms (Fig. 3). 


\section{Changes in phytoplankton community structure at three touristic sites at western Alexandria Beach}

However, the three biological parameters (phytoplankton diversity, richness and evenness) showed different temporal pattern. The absolute values of diversity index displayed bimonthly variations from 0.56 nats in July to 2.54 nats in March (Fig. 5), whereas the evenness fluctuated from 0.29 in September to 0.82 in May and the richness from 0.22 in July to 5.19 in November (Fig. 3).

The phytoplankton abundance was very low in the village, ranging from $85 \mathrm{unit} /$ Liter in July to $10 \times 10^{3}$ unit/Liter in September (Fig. 5), with an averge of $3 \times 10^{3}$ unit/Liter (Table 3). Rhizosolenia setigera was responsible for a small peak in September (76.62\% to total count).

The total chlorophyll- $a$ showed narrow temporal variations from 0.27 $\mu \mathrm{g} / \mathrm{L}$ in May to $1.78 \mu \mathrm{g} / \mathrm{L}$ in September. However, the net chlorophyll contributed by $83.50 \%$ to the total chlorophyll (Fig. 6).

\section{Marabella touristic village site:}

The results of the physico-chemical parameters of water samples collected at the Marabella village site were not greatly different from those of Marakia village site (Table 1). Water temperature ranged from 16.61 to $30.00^{\circ}$ C. Water salinity fluctuated betweeb 38.61 and 39.95PSU. Water transparency varied from 100 to $300 \mathrm{~cm}$. $\mathrm{pH}$ values lied on the alkaline side $(7.98-8.27)$. Dissolved oxygen concentration ranged from 4.25 to $19.55 \mathrm{mg} \mathrm{O}_{2} / \mathrm{L}$ with an average of $10.07 \mathrm{mg} \mathrm{O}_{2} / \mathrm{L}$. The nutrient salts and the oxidizable organic matter were relatively low similarly as at the Marakia village site (ammonia: $0.50-10.48$ $\mu \mathrm{M} / \mathrm{L}$, nitrite: $0.00-1.03 \mu \mathrm{M} / \mathrm{L}$, nitrate: $0.44-6.88 \mu \mathrm{M} / \mathrm{L}$, phosphate: $0.00-1.92$ $\mu \mathrm{M} / \mathrm{L}$, silicate: $0.23-1.20 \mu \mathrm{M} / \mathrm{L}$ and oxidizable organic matter: $0.16-6.44 \mathrm{mg} / \mathrm{L}$ ).

Phytoplankton community at the Marabella village Site was less diversified compared with the previous sites, comprising a total of 54 taxa (Table 2), that included 30 taxa purely marine and 24 freshwater and brackish forms (Fig. 4). Bacillariophyceae was the most diversified group (18 genera, 34 taxa) followed by Dinophyceae ( 7 genera, 9 species), Chlorophyceae (5 genera, 5 species), Cyanophyceae ( 3 genera, 3 species), Euglenophyceae (1 genus, 2 species) and Silicoflagellates (1 genus, 1 species) (Table 3). Number of species varied between three taxa in July to 24 taxa in January (Fig. 3). Bacillariophyceae predominated during the study period except in September, where chlorophytes formed $99.53 \%$ of total count (Fig. 2).

Diversity and richness indices ranged between 0.03 nats and 0.47 in September to 2.79 nats and 3.40 in January (Fig.3 \&5), whereas the evnness values fluctuated between 0.01 in September to 0.83 in July (Fig. 3 ).

Phytoplankton abundance fluctuated between 34 unit/L (July) and $2.7 \times 10^{6}$ unit/Liter in September due to the overwhelming of Carteria sp. contributing by $99.53 \%$ of the total count (Fig. 5).

Similar to the other sites, the total chlorophyll- $a$ in Marabella village site attained low concentrations varying from nil in July to $1.78 \mu \mathrm{g} / \mathrm{L}$ in September, coinciding with the phytoplankton peak during this month. On the other hand, the net chlorophyll $<20$ contributed by $72.93 \%$ of the total chlorophyll (Fig. 6). 


\section{DISCUSSION}

The Mediterranean Sea is known to be oligotrophic (Ediger \& Yilmaz, 1996), it is rich in dissolved oxygen and poor in nutrients. Oligotrophic status increases from west to east (Zenetos et al., 2005). The pattern may have been altered in the last few years, because of changes in water characteristics, perhaps in response to human activities (Turley, 1999). Urbanization and tourism result in pollution by sewage along the coastal zone, increasing the seawater temperature and salinity in both the eastern and western basins (Bethoux \& Gentili, 1996 and 1999; Roether, et al., 1996).

The area under investigation lies to the southeastern Mediterranean coast, under the influence of different kinds of wastewater discharges. El-Mohandessin village site receives huge amount of industrial effluents from petroleum and power plant, along with the sewage discharges. At Marakia and Marabella villages, sites are subjected to sewage discharges but no stress of industrial discharges.

Naubaria site receives agriculture and domestic wastewaters at the connecting point between the Naubaria Canal and the sea (mean salinity 9.7 PSU). The effect of freshwater discharged was insignificant at stations II-IV (mean salinity 36.8 PSU). The surface current in the canal is usually directed towards the sea, supposing gradual successive increase of phytoplankton abundance and biomass (chlorophyll- $a$ ). A reversed subsurface current carries seawater into the canal (Gharib and Dorgham, 2003). Surface water salinity of the Mediterranean Sea fluctuated between 36.2 and 39.1 PSU (EEA, 1999), higher values of water salinity were recorded at Marakia and Marabella tourism villages sites. This may be resulting from the fact that evaporation rate exceeds precipitation and frsihwateer runoff and therefore higher salt content of water results (Stuart and Tsimplis, 2003). The $\mathrm{pH}$ values at Naubaria region are on the alkaline side, reflecting the effect of photosynthetic activity, causing more consumption of carbon dioxide and the rise of $\mathrm{pH}$ level at the surface water and decrease towards near bottom layer with the decrease in photosynthetic activity (Abdalla et al., 1995). Oxygen content reached oversaturation sometimes (19.55 $\left.\mathrm{mgO}_{2} / \mathrm{L}\right)$. The concentration of dissolved inorganic nitrogen showed high noticeable values at station I of Naubaria site $(28.7-76.0 \mu \mathrm{M} / \mathrm{L})$. The effect of discharged water rich with nutrient salts decreased seawards. At the three tourism villages sites, dissolved inorganic nitrogen showed less concentrations, having averages of $12.2,7.4$, and $7.2 \mu \mathrm{M} / \mathrm{L}$ for El-Mohandessin, Marakia and Marabella, respectively. Ammonium has been recognized as important alternative nitrogen source for various aquatic plants and in most environments may be assimilated in preference to nitrate (Wafar et al., 1986). Average annual concentration of nitrate was $10.1 \mu \mathrm{M} / \mathrm{L}$ at Naubaria site decreased to $8.23 \mu \mathrm{M} / \mathrm{L}$ in El-Mohandessin village site. The lowest nitrate concentrations were observed in Marakia site $(2.10 \mu \mathrm{M} / \mathrm{L})$ and Marabella site $(2.87 \mu \mathrm{M} / \mathrm{L})$. The same pattern was observed for phosphate and silicate concentrations, where lowest average 


\section{Changes in phytoplankton community structure at three touristic sites at}

western Alexandria Beach

concentrations were recorded in Marakia and Marabella (0.6-0.8; 0.5-1.3 $\mu \mathrm{M} / \mathrm{L})$ for phosphate and silicate, respectively. The 208 identified phytoplankton taxa recorded in the investigated area, included species previously appeared in different parts of the eastern coast of the Mediterranean Sea (Dowidar, 1974; Lakkis and lakkis, 1980 and Polat et al., 2000; Polat and Isk, 2002; Gharib and Dorgam, 2006 and Gharib, 2006; Shams EL Din and Dorgham, 2007). Naubaria site was more diversified (176 taxa) followed by El-Mohandessin (90 taxa) and marakia sites (83 taxa), while the lowest number of species was recorded in Marabella site (54 taxa). The large number of diatoms and freshwater species are responsible for the higher phytoplankton diversity in Naubaria site. This agrees with the observations of Margalef (1960) and Kingston et al. (1983) who pointed out that diversity would be high when two different communities mix together. The structure of phytoplankton community showed high variations among different locations and different seasons, except the drop in counts during March-May. Furthermore, the occurrence of $(13 \%)$ of phytoplankton assemblages during the investigated period indicated a type of stability in a part of the community which have capability to tolerate different kinds of pollutants, in addition to the variations of the environmental conditions regardless of their numerical densities.

At Naubaria site, the influence of freshwater discharged from Naubaria Canal was significant at station I, where the algal community structure was composed mainly of freshwater and brackish water forms. Bacillariophyceae was the dominant group (63.4\%) followed by Cyanophyceae $(25.1 \%)$, the density of which increased towards the sea becoming the dominant (92.499.4\%). Cyclotella kütziginiana and Rhizosolenia fragellissima were responsible for the peaks in July and September. However, Palmer (1969) included the genus Cyclotella among the indicators of organic pollution. Halim et al. (1980) mentioned that $R$. fragellissima is considered intolerant to pollution and that it is restricted to offshore areas, while Abdallah et al. (1995) recorded this species as tolerant to pollution.This species was also considered as indicator of eutrophication (Zaghloul, 1994a). The same species was recorded as one of dominant species in Abu-Qir Bay and was considered as indicator of pollution (El-Sherif and Mikhail, 2003).

Among the three touritic villages phytoplankton abundance showed relatively big differences, whereas the highest count (average $142 \times 10^{3}$ unit/L) was observed in El Mohandessin village site, during November. Skeletonema costatum was an active contributor in November peak. The lowest counts were observed in Marakia and Marabella sites with the exception of Rhizosolenia setigera, which formed $76.6 \%$ of the total count $\left(10 \times 10^{3}\right.$ unit/L) recorded in September (Marakia site) and the absolute dominance of the green alga Carteria sp. $(99.53 \%)$ of the total count $\left(2.7 \times 10^{6}\right.$ unit/L) in September (Marabella site). However, the genus Carteria is not listed among harmful algae and the algal bloom do not pose a significant threat to human health or aquatic recreational 
activities, but did have a direct aesthetical impact due to watercraft discoloration. Extended outbreaks of algae can potentially impact the ecological balance to the detriment of the existing macrophytes (Ecological Engineering, 2005). The genus Carteria was previously recorded in the Mediterranean Egyptian waters (El-Sherif and Mikhail, 2003), despite its habitat as pure freshwater form (Reynolds, 2006), and was found to live in sewage waters and wastewater treatment ponds (Chapman et al., 1957), which may indicate that the genus is an indicator for sewage water and has adapted to live in Marabella village site in salinity ranging from 38.61 to $39.95 \mathrm{PSU}$, where it attained a peak of $2.7 \times 10^{6}$ unit/L. However, Carteria was recorded in Lake Edku during year 2000 accompanied by high nutrient salts (Zaghloul and Hussein, 2000).

A focus was stressed on El-Mohandessin village site because it received huge amount of industrial effluents from a Petroleum Company and hot water from a power plant along with the sewage discharges. A major problem of power plants water effluent discharge is thermal pollution that loads to a decrease in dissolved oxygen and an increase in water temperature. Increases in temperature affect phytoplankton assemblage by eliminating stenothermal forms and increasing eurythermal species (Naylor, 1965), thereby altering phytoplankton community structure. No detectable rise in water temperatures was recorded during sample collections, this may be due to cooling due to rapid mixing resulting in little impact (Davis et al., 2001). This may explain the overwhelming of the neritic, euryhaline and eurythermal Skeletonema costatum, which formed $98.5 \%$ of the total count, and it could grow quickly under eutrophic conditions (Huang et al., 2004). The presence of Skeletonema costatum reflected the effect of freshwater discharge (Abdallah et al., 1995; El-Sherif and Mikhail, 2003; Gharib, 2006; Nassar and Shams El-Din, 2006) and the high amount of oxidizable organic matter (Zaghloul, 1995). The occurrence of Skeletonema costatum is considered as indicator of eutrophication (Smayda 1965) and pollution (Mihnea, 1985). On the other hand, Umamaheswara Rao and Mohanchand (1988) indicated that Skeletonema costatum is a pollution tolerant and bloom forming species. However, Skeletonema costatum was previously recorded as blooming species causing sometimes red tide in the Mediterranean Egyptian waters, in mouth of Damietta branch (Halim, 1960), Eastern Harbour (El-Maghraby and Halim, 1965; Zaghloul, 1995 and Mikhail, 2001), Mex Bay (Mikhail, 1997), El-Dekhaila Harbour (Ismael and Abdel Aziz, 2003) and in Abu-Qir bay (El-Sherif and Mikhail, 2003). They recorded the species in a wide range of salinity $(0.17-39 \mathrm{PSU})$, and temperature $\left(12.3-30^{\circ} \mathrm{C}\right)$, which may indicate its ability to live in different ecological conditions as a tolerant species.

A reduction in dissolved oxygen reaching $4.01 \mathrm{mg} / \mathrm{L}$ in May was observed in El Mohandessin, accompanied with abnormal values of oxidizable organic matter $(116 \mathrm{mg} / \mathrm{L})$ and reactive silicate $(83.11 \mu \mathrm{M} / \mathrm{L})$, where Euglenophytes and chlorophytes dominated the algal community (44.8 and 
$20.1 \%$ of the total count, respectively), in water salinity of 38.7 PSU. During January when oxidizable organic matter and dissolved inorganic nitrogen were $12 \mathrm{mg} / \mathrm{L}$ and $15.3 \mu \mathrm{M} / \mathrm{L}$, respectively, euglenophytes increased to form $69.3 \%$ of the total community. The prevailing of the euglenophycean (Euglena) in May and January, acted as the most tolerant genus of organic pollution (Danilov and Ekulend, 2001 and Forray, 2002). This is in agreement with Rossmann et al., (1980) who found that at elevated temperature, phytoplankton assemblages exhibited a decrease in species diversity, seen as a shift from a diatom dominated assemblage to one dominated by green algae or euglenophytes. The results suggest that El Mohandessin village site lies on multiple pollution, which are responsible for alteration of the ecosystem structure, but the excellent wind driven mixing kept the water well aerated, thus reducing the severe pollution stress by dispersing the organic (4.01- $13.57 \mathrm{mg} \mathrm{O}_{2} / \mathrm{L}$ ) and other pollutants.

The eastern Mediterranean waters was oligotrophic i.e. chlorophyll- $a$ was $<0.5 \mu \mathrm{g} / \mathrm{L}$ (Robarts et al., 1996). The oligotrophy of the Mediterranean Sea is reflected in the size spectrum of phytoplankton, generally shifted towards small dimensions. The present study indicated that the relative contribution of nanophytoplankton was high. The concentrations of Chlorophyll- $a$ varied from 0.46 to $19.82 \mu \mathrm{g} / \mathrm{L}$ with an average of $2.66 \mu \mathrm{g} / \mathrm{L}$ (Naubaria site), from 0.46 to $8.26 \mu \mathrm{g} / \mathrm{L}$, with an average of $2.37 \mu \mathrm{g} / \mathrm{L}$ (El Mohandessin site), from 0.27 to $1.78 \mu \mathrm{g} / \mathrm{L}$, with an average of $0.92 \mu \mathrm{g} / \mathrm{L}$ (Marakia site), and between nil to 1.78 $\mu \mathrm{g} / \mathrm{L}$ with an average of $0.77 \mu \mathrm{g} / \mathrm{L}$ (Marabella site). According to the classification of Wetzel (2001), Naubaria site and El-Mohandessin lied between meso- to oligo trophic status, but the two tourism villages sites were mainly oligotrophic, denoting to no environmental problems encountered (Walker, 1985). The temporal variations of chlorophyll- $a$ did not coincide with that of the cell count. For example, the high concentrations of chlorophyll- $a$ recorded in July were not coinciding with the peaks recorded in different locations of the study area. This can be attributed to the higher portion of pico- and nanoplankton which constituted 83.7, 72.8, 83.5 and $72.9 \%$ of the total averge of biomasses in Naubaria, El-Mohandessin, Marakia and Marabella sites, respectively. Thus the high percentage of nanoplankton was reflected the effective contribution in the phytoplankton biomass. The role of nano- and picoplankton in phytoplankton biomass was reported in the Egyptian coastal waters (Gharib and Dorgham, 2006; Shams El Din and Dorgham, 2007). Contamination of water with petroleum oil may benefit the microphytoplankton, inhibits the growth of nanoplankton and does not affect significantly the picoplankton as reported by Zhu et al. (2000). This conclusion agrees with the present result in El Mohandessin site during the period March to July when nanoplankton contributed $<40 \%$ of the total biomass.

The diversity index may be used as suitable criteria for water quality (Balloch et al., 1976). The values of diversity index are related to evenness, while they are not affected by the species number (richness) (Reed, 1978; Ismael 
and Dorgham, 2003). In contrast, the present study provides a clear relation between diversity index, degree of dominance, species richness and phytoplankton abundance. For example, the lowest diversity (0.03nats) was recorded in Marabella village site in September with absolute predominance of Carteria sp. and low number of species (8), while the highest diversity index (2.99 nats) appeared at staion I in November at Naubaria site, due to multidominance and high number of species (38). Gharib and Dorgham (2003) reported similar observations in the west of Naubaria Canal. The values of diversity index were affected also by the environmental conditions; the highest values appeared at the outfall of Naubaria Canal (Station I), which was pronouncedly affected by land- based effluents. Rao and Mohanchand (1988) stated that reduction in the number of dominant species and species diversity and the increase in cell count of one or two resistant algae were some of the changes observed in the phytoplankton populations in domestic and industrially polluted environments. The values of diversity index in the area under investigation (0.03-2.99 nats) are nearly close to those found (1-2.5 nats) by Margalef (1964, 1978 ) in the active growing coastal populations. Approximately, similar values (0.8-2.4 nats) were reported by Ismael and Dorgham (2003) in Dekhaila Harbour (west of Alexandria). It should be mentioned that the values of phytoplankton diversity index obtained during the present study are underestimated, since nanoand picoplankton cells were not considered in the count. The values of diversity index in the present study, according to Hendys classification related to moderately or slightly polluted waters (Hendy, 1977). Hence, lower species diversity and proliferation of certain species of Chlorophyceae and Euglenophyceae and other freshwater organisms could be used as a measure to detect moderate environmental disturbances prevailing El Mohandessin and Marabella villages sites. Whereas, higher diversity of diatoms and dinoflagellates at Marakia village site suggests free conditions, which are favourable for healthy community growth.

\section{CONCLUSION}

The dominance of freshwater and brackish forms and the dense blooming of few of them accompanied with low diversity during the course of the study reflected the effect of wastewater effluent disharge at El-Mohandessin and Marabella villages sites which should be kept continuously under control, whereas the environmental conditions prevailing at Marakia village site, were favourable for healthy community growth.

\section{ACKNOWLEDGEMENT}

We are greatly indebted to Prof. Dr. Zeinab Mahmoud El Sherif, Supervisor of the project of the study of the effect of industrial activities, marine transport and tourism on physico-chemical and biological characteristics of water and fisheries groups in wester Alexandria, funded by National Institute of 


\section{Changes in phytoplankton community structure at three touristic sites at western Alexandria Beach}

Oceanography and Fisheries, who helped and encouragedus to acconplih this work.

\section{REFERENCES}

Abdallah, R. R.; Zaghloul, F. A. and Hussein, N. R. (1995). A statistical modeling of phytoplankton eutrophication in the Eastern Harbour, Alexandria, Egypt. Bull. Nat. Inst. Of Oceanogr. \& Fish., A.R.E. 21 (1): $125-146$.

Abdel Aziz, N. E. (2005). Short term variations of zooplankton community in the West Naubaria Canal, Alexandria, Egypt. Egyptian J. of Aquatic Research. 31(1): 119-133.

American Public Health Association (APHA), (1995). Standard Methods for the Examination of water and wastewater "19 ${ }^{\text {th }}$ Edition prepared and published jointly by American Public Health Association, American water works Association, and water Environment Federation. Fifteenth sheet, N.W. Washington D.C. 2005. 1015 pp.

Balloch, D. ; Davies, C. E. and Jones, E. H. (1976). Biological assessement of water quality in three British rivers: The North Esk (Scotland), the Ivel (England) and the Taff (Wales), Water Pollution Control, 75: 92-114.

Bethoux, J. P. and Gentili, B. (1996). The Mediterranean Sea, coastal and deepsea signatures of climatic and environmental changes. J. Mar. Syst., 7:383-394.

Bethoux, J. P. and Gentili, B. (1999). Functioning of the Mediterranean Sea: past and present changes related to freshwater input and climate changes. J. Mar. Syst., 20:33-47.

Chapman, V. J.; Thompson, R. H. and Secar, M. (1957). Check list of freshwater Algae of New Zealand. Trans. R. Soc. N.Z. 84 (4): 695-747.

Cupp, E. E. (1943). Marine plankton diatoms of the west coast of North America. Bull. Scipps Inst. Oceanogr., 5 (1):1-238.

Ecological Engineering (2005). Ecological and Human Health Risk Assessment for Lake Wendouree. In Lake Wendouree Water Supply Investigation Report 29 November 2005, 84pp.

EL-Maghraby, A. M. and Halim,Y. (1965). A quantitative and qualitative study of the plankton of Alexandria waters. Hydrobiologia, 25(1-2): 221-238. 
EEA, (1999). State and pressure of the marine and coastal Mediterranean environment. Environmental assessment Series, 5.

Ediger, D. and Yilmaz, A. (1996). Characteristics of deep chlorophyll maximum in the northeastern Mediterranean with respect to environmental conditions. J.Mar. Systems, 9: 291-303.

Ellis, M. M.; Westfall, B. A. and Ellis, D. M. (1946). Determination of water quality "Fish and wildlife service, Research report 9: 122p.

El- Sherif, Z. and S. K., Mikhail.(2003). Phytoplankton dynamics in the southwestern part of Abu Qir Bay, Alexandria, Egypt. Egypt. J. Aquat. Biol. \& Fish., 7 (1): 219- 239.

Danilov, R. A. and Ekulend, N. G. A.(2001). Using the green flagellate Euglena gracilis Klebs as physiological Dosimeter: is a long-term Bioassay more significant than a short-term one? Turk. J. Bot. 25:43-44.

Davis, N. ; Koslowsky, S.; Foster, M. and York, R. (2001). Biological Resources, P.231-258. In: Final Staff Assessment -Huntigton Beach Generating Station Retool Project. California Energy Comission, Sacraments.

Dowidar, N. M. (1974). The phytoplankton of the Mediterranean waters of Egypt. A checklist of species recorded. Bull.In.Oceanography Fish., 4: 319-344.

Forray, F. (2002). Environmental pollution in the Aries River catchement basin. Case study: Rosia Montana Mining Exploitation. Studia universitatis Babes-Bolyai, Geologia, Special ISSUE (1):189-198.

Gharib, S.M. and Dorgham, M.M. (2003): Weekly variations of phytoplankton community in the west Naubaria Canal, Alexandria, Egypt. J. Egypt. Acad. Soc. Environ. Develop., (D-Environmental studies) 4(3): 201218.

Gharib, S. M. and Dorgham, M. M. (2006). Eutrophication stress of phytoplankton community in the western Harbour of Alexandria, Egypt. Int.Journal of Ocean\&Oceanog., 1(2):261-273.

Gharib, S..M. (2006). Effect of freshwater flow on the succession and abundance of phytoplankton in Rosetta Estuary, Egypt. International Journal of Ocean and Oceanography, (1): 2 (under press). 
Grasshoff, K. (1976). Methods of seawater analysis, Verlag Chemie Weinheim, New York. 317 pp.

Halim, Y. (1960). Observations on the Nile Bloom of Phytoplankton in the Mediterranean. J.du Conseil, 26 (I): 57-67.

Halim, Y.; Khalil, A. and Handhal, A. (1980). Diatoms flora of an eutrophic bay, the Eastern Harbour of Alexandria. Acta Adriatica, 21 (2): 271-298.

Heip, C. (1974). A new index measuring eveness. J.of marine biological association of the U. K., 54: 555-557.

Hendy, N. I. (1977). In Proc. $4^{\text {th }}$ Symposium. Recent and fossil Marine Diatoms (University of Oslo, Nova Hedwig), cited by Tiwari, L.R. and Nair.V.R. (1988) Ecology of phytoplankton from Dharmar creek, west coast of India. Indian J. Mar. Sci. 27:302-309.

Heurk, V. H. (1962): A treatise on the Diatomaceae. Wheldon and Wesly, Ltd. And Verlag. G. Gamer, Berlin. 558 pp.

Huang, L.; Jian, W.; Song, X.; Huang, X.; Liu, S.; Qian, P.; Yin, K. and Wu, M. (2004). Species diversity and distribution for phytoplankton of the Pearl River estuary during rainy and dry seasons, "Marine Pollution" Bull., 49: 30-39.

Ismael, A. A. and Abel Aziz, N. E. (2003). Selective grazing on phytoplankton in El-Dekhaila harbour, Alexandria. Marine life, 13(1-2):21-30.

Ismael, A. A. and Dorgham, M. M.(2003). Weekly variations of phytoplankton community in the west Naubaria Canal, Alexandria, Egypt. J. Egypt.Acad.Soc.Environ.Develop. (D.Environmental Studies), 4(3):201218.

Kingston, J. C. ; Lowe, R. L.; Stoermer, E. F. and Ladewski, T. B. (1983). Spatial and temporaldistribution of benthic diatoms in northern Lake Michgan . Ecology, 64:1566-158.

Lakkis, S. and Novel-Lakkis, V. (1980). Composition, annual cycle and species diversity of the phytoplankton in Lebanese coastal water. J. Plank. Res. $3(1): 123-135$.

Lebour, M. V. (1925). The Dinoflagellates of Northern seas published by Marine Biological association of the United Kingdom $250 \mathrm{pp}$. 
Margalef, D. R. (1960). Temporal succession and spatial heterogeity in phytoplankton. In: BUZZATITRAVERSE. A. A.(ed.): Perspectives in marine Biology: 323-343pp.

Margalef, D. R. (1964). Correspondance between the classic types of lakes and the structural and dynamic properties of their populations. Verh. Int. Verein. Limnol., 15:169-175.

Margalef, D. R. (1968). Perspectives in Ecological Theory University of Chicago Press, Chicago, IL, 111 pp.

Margalef, D. R. (1978). Life forms of phytoplankton as survival alternatives in an unstable environment. Oceanol. Acta, 1:493-509.

Mihnea, P. E. (1985). Effect of pollution on phytoplankton species. Rapp. Comm. Int. Mer. Medit. 29 (9): 85-88.

Mikhail, S. K. (1997). Ecological studies of the phytoplankton in Mex Bay. Ph.D. Thesis, Alexandria Univ., 266pp.

Mikhail, S. K. (2001). Phytoplankton variability in the Eastern Harbour of Alexandria during 2000. Bull. Nat. Oceanogr. \& Fish., A. R. E., 27: 3252.

Mizuno, T. (1990). Illustrations of the freshwater plankton of Japan. $9^{\text {th }}$ Printing, Horkush Publishing Co., Lt. Japan, 353 pp.

Nassar, M. Z. and Shams El-Din, N. G. (2006). Seasonal dynamics of phytoplankton community in the Bitter Lakes and Lake Temsah. Bull. Nat. Inst. of Oceanogr. \& Fish., A.R.E. (32) No. 1.

Naylor, E. (1965). Effect of heated effluents upon marine and estuarine organisms. Advances in Marine Biology., 3:63-103.

Palmar, C. M. (1969). A composite rating of algae tolerating organic pollution J. of Phycol., 5: 78-82.

Peragallo, M. and Peragallo, M. M. H. (1897-1908). Diatomeés Marines de France et des Districts Maritimes Voisins, 236 pp.

Polat, S.; Sarihan, E. and Koray, T. (2000). Seasonal changes in the phytoplankton of the Northeastern Mediterranean (Bay of Iskenderun). Turk. J. Bot. 24:1-12. 
Polat, S. and Isik, O. (2002). phytoplankton distribution, diversity and nutrients at the north-eastern Mediterreneancoast of Turkey (KaratasAdana).Turk. Journal of Botany., 26:77-86.

Rao, M. U. and Mohancland, V. (1988). Water quality characteristies and phytoplankton of polluted visa phapatnam harbour. Marine Environmental Research 25: 23-43.

Reynolds, C. S. (2006). The Ecology of Phytoplankton. Published in the United States of America by Cambridge University Press, New York. 550pp.

Robarts, R. D.; Zohary, T.; Waiser, M. J. and Yacobi, Y. Z. (1996). Bacterial abundance, biomass, and production in relation to phytoplankton biomass in the Levantine Basin of the southeastern Mediterranean Sea, Mar. Eco. Progress Series, 137:273-281.

Roether. W.; Manca, B.B.; Klein, B.; Bregant, D. (1996). Recent changes in eastern Mediterranean deep waters. Science, 271:333-335.

Rossmann, R. ; Chang, W.; Damaske, L. and Yocum, W. (1980). Entrainement ofphytoplankton at the Donald C. Cook Nuclear Plant-1977. p180.

Reed, C. (1978). Species diversity in aquatic micro-ecosystem. Ecology, 59:481488.

Shams El Din, N. G. and Dorgham, M. M. (2007). Phytoplankton community in Abu-Qir Bay as a hot spot of the southeastern Mediterranean coast. Egypt. J. of Aquatic Resear., 33(1): 163-182.

Shannon, C. E. and Weaver (1963). The mathematical theory of communication Univ. of Tllinoris. Press., Urbana 117pp.

Smayda, T. J. (1965). A quantitative analysis of the phytoplankton of the Gulf of Panama III. On the relationship between $\mathrm{C} 14$ assimilation and the diatom standing crop. Inter. Amer. Tropical Tuna Comiss. Bull., 9 (7): 467-531.

Sournia, A. (1968). Diatomeés planctoniques da canal de Mozambique et de Lille Maurice, ORSTOM Mein, 31: $120 \mathrm{pp}$.

Sournia, A. (1986). Atlas du phytoplankton Marin, Volume 1: Introduction, Cyanophyceés, Dictychophyceés et Radiophyceés 216 pp. 
Stuart, C. and Tsimplis, M. N. (2003). Temperature and salinity trends in the upper waters of the Mediterranean Sea as determined from the MEDATLAS dataset. .J. of Continental shelf Resear. 23 (16): 15071522.

Tikkanen, T. (1986). Kaviplanktonopas, Helsiniki. 278 pp.

Turley, C. M. (1999). The changing Mediterranean Sea- a sensitive ecosystem? Progr. Oceanogr., 44: 387-400.

Wafar, M. V. M.; Wafar, S. and Devassy, V. P. (1986). Nitrogenous nutrients primary production in a tropical oceanic environment. Bull. Mar. Sci., 38 (20):273-284.

Walker,W. W. (1985). Staistical bases for mean chlorophyll- $a$ criteria. Lake and Reservoir Management: Practical Applications. Proc. $4^{\text {th }}$ Annual Conference, North American Lake Management Society, McAfee, New Jersey, 55-62.

Wetzel, R. G. (2001). Limnology: Lake and River ecosystems. Academic Press, San Diego.1006pp.

Zaghloul, F. A. and Hussein, N. R. (2000). Impact of pollution on phytoplankton community structure in lake Edku, Egypt. Bull. Nat. Inst. of Oceanogr. And Fish. 26: 297-318.

Zaghloul, F. A. (1994a). Phytoplankton dynamics in the Western Harbour of Alexandria, Egypt. Bull. Nat. Inst. of Oceanogr. And Fish. 20 (2): 107117.

Zaghloul, F. A. (1995). Comparative study of phytoplankton production, composition and diversity index in the Eutrophic Eastern Harbour of Alexandria, Egypt. Bull. of High inst.of Public health, 25(3):665-678.

Zenetos, A.; Sioko, I. and Gotsis, O. (2005). The Mediterranean Sea. European Environment Agency, 22pp.

Zhu, M.; Chen, I. and Lu, R. (2000). Effect of oil pollution on marine pelagic ecosystem. Research report from the National Institute for Environmental studies, Japan. 